Canadian University Music Review

Canadian University Music Review

Revue de musique des universités canadiennes

\title{
Écriture tonale et perspectives nouvelles de l'harmonie fauréenne
}

\section{Sylvain Caron}

Volume 22, numéro 2, 2002

URI : https://id.erudit.org/iderudit/1014506ar

DOI : https://doi.org/10.7202/1014506ar

Aller au sommaire du numéro

\section{Éditeur(s)}

Canadian University Music Society / Société de musique des universités canadiennes

\section{ISSN}

0710-0353 (imprimé)

2291-2436 (numérique)

Découvrir la revue

Citer cet article

Caron, S. (2002). Écriture tonale et perspectives nouvelles de l'harmonie fauréenne. Canadian University Music Review / Revue de musique des universités canadiennes, 22(2), 48-76. https://doi.org/10.7202/1014506ar

\section{Résumé de l'article}

Dans une analyse orientée vers des applications en écriture, l'auteur démontre comment Fauré intègre à la tonalité classique une conception nouvelle des fonctions harmoniques. Si la dominante conserve son rôle structurant en contexte cadentiel, la multiplication des dominantes défonctionnalisées rendent équivoques les progressions en cours de phrase. Comme autres procédés, on observe par endroits un usage « autonome » de la modalité, la séquence harmonique, l'harmonie des médiantes et un contrepoint qui prédomine de plus en plus sur l'harmonie; ces techniques viennent modifier considérablement, mais de l'intérieur, les caractéristiques du langage tonal. Au lieu de définir ces nouvelles caractéristiques par rapport à la tonalité, il est préférable de parler de nouvelles fonctions tonales. Celles-ci deviennent parties intégrantes du langage car elles conditionnent la structure harmonique globale.
All Rights Reserved (C Canadian University Music Society / Société de musique des universités canadiennes, 2003
Ce document est protégé par la loi sur le droit d'auteur. L'utilisation des services d'Érudit (y compris la reproduction) est assujettie à sa politique d'utilisation que vous pouvez consulter en ligne.

https://apropos.erudit.org/fr/usagers/politique-dutilisation/ 


\title{
ÉCRITURE TONALE ET PERSPECTIVES NOUVELLES DE L'HARMONIE FAURÉENNE
}

\author{
Sylvain Caron
}

Dans les pratiques universitaires actuelles en milieu francophone, l'enseignement de l'écriture vise essentiellement la connaissance du langage classique ${ }^{1}$, et ne touche que bien sommairement à l'harmonie tonale postromantique. Les traités d'harmonie publiés en français sont d'ailleurs assez anciens (comme celui de Kœchlin, qui date de $1928^{2}$ ), ou ne font que reprendre ces informations en les classifiant plus synthétiquement ${ }^{3}$. Écrits au moment où l'harmonie s'engageait dans une voie relativement nouvelle, ces ouvrages souffrent de l'absence de recul, élément souvent nécessaire à l'explication structurelle du langage. Il est vrai que le Harmonielehre de Schoenberg offre un regard plus moderne ${ }^{4}$, mais l'objet de son étude porte essentiellement sur le langage classique, et non sur les nouvelles perspectives harmoniques du début du XX siècle. Certes, il existe plusieurs publications qui traitent de manière analytique de l'évolution de la tonalité à partir de la fin du XIXe siècle d'équivalent français du magistral Contemporary Harmony de Ulehla ${ }^{6}$, sorte de traité d'écriture pour l'époque postromantique. Outre la codification et la classification des procédés, l'auteure oriente ses analyses vers des considérations utiles pour la réalisation - comme la construction des mélodies et des motifs, la conduite des voix ou l'emploi des 12 degrés chromatiques dans un contexte diatonique - et propose des exercices d'écriture à la fin des chapitres.

Parmi la soixantaine de compositeurs étudiés par Ulehla, on retrouve la plupart des grandes figures, dont Brahms, Wagner, Debussy, Ravel, Stravinsky et Barber. Il y a pourtant une omission de taille : Gabriel Fauré. Or, afin de comprendre par l'écriture comment l'harmonie s'est émancipée des cadres classiques, l'étude de Fauré est d'un grand intérêt, puisque le compositeur est au cœur des profondes transformations qui surviennent entre Gounod et De-

$1 \mathrm{~J}$ 'emploie le mot " classique " dans le sens d' " usuel ", à l'intérieur de ce que Carl Dahlhaus nomme La Tonalité harmonique (Liège : Mardaga, 1993) et qui correspond approximativement à la période comprise entre 1700 et 1880.

2Charles Kœchlin, Traité de l'harmonie (Paris : Éditions Max Eschig, 1928).

3 Voir, par exemple, Marcel Bitsch, Précis d'harmonie tonale (Paris : Leduc, 1957).

4 Arnold Schoenberg, Harmonielehre (Vienne : Universal Edition, 1911); trad. de l'allemand par Gérard Gubisch sous le titre de Traité d'harmonie (Paris : Jean-Claude Lattès, 1983).

5 Voir, par exemple, Walter Piston et Mark DeVoto, «After Common Practice », dans Harmony, 5 éd. (New York : Norton, 1987), 457-542; Deborah Stein, "The Expansion of the Subdominant in the Late Nineteenth Century ", Journal of Music Theory 27 (1983) : 153-80.

6 Ludmila Ulehla, Contemporary Harmony-Romanticism through the Twelve-Tone Row (New York : Advance Music, 1994). 
bussy. Formé à l'école de Niedermeyer, Fauré s'inspire d'une part des classiques et des premiers romantiques allemands - tous représentatifs de la tonalité " classique » - et d'autre part de la musique religieuse, avec les maîtres de la Renaissance et le plain-chant - qui défendent une vision prétonale de l'harmonie. C'est notamment en raison de cette double filiation, avec ses conséquences sur l'évolution de la musique française de la période de Debussy et de Ravel, que le langage de Fauré présente un intérêt pédagogique.

Par ailleurs, j'adopte ici une perspective différente de celle des classes d'écritures en France, où les réalisations de chants donnés dans le style de Fauré sont courantes. Au lieu de viser l'apprentissage de certaines progressions-types - notamment des septièmes de dominantes non fonctionnelles -, l'imitation stylistique ou la reconstitution d'un vocabulaire, dans la lignée des travaux de Françoise Gervais ${ }^{7}$, je cherche plutôt à expliquer comment Fauré renouvelle les fonctions harmoniques tout en conservant l'essentiel des cadres de la tonalité classique. Edward R. Phillips a d'ailleurs déjà démontré, par l'analyse schenkérienne, que le langage de Fauré procède par ornementation des structures classiques ${ }^{8}$. Sans nier l'intérêt de l'analyse schenkérienne, qui rattache Fauré à la tonalité, je crois tout de même que l'intérêt réside d'abord dans l'étude de certains des procédés nouveaux qu'il a développés : en quoi consiste le "flou harmonique », parfois si déconcertant, qui se déploie entre les indicateurs tonaux?

Essentiellement, Fauré ne détruit pas la tonalité, mais il en élargit le cadre de l'intérieur même du système au moyen de six procédés : l'équivoque, la modalité autonome, la séquence harmonique, l'harmonie des médiantes, le chromatisme des fonctions et le contrepoint directionnel. La notion d'équivoque, introduite par Ken Johansen ${ }^{9}$, repose sur un jeu équilibré d'attentes résolues ou non. Lorsque l'attente n'est pas résolue, c'est que l'accord est réinterprété en fonction d'un nouveau contexte. L'accord qui crée le plus d'attente et qui, par conséquent, peut entraîner le plus de surprises, est celui de dominante, puisqu'il appelle une résolution à la tonique. En outre, nombre d'équivoques ne sont que graphiques, donc sans effet sur l'audition, étant causées par l'enharmonie. Si la cohabitation de la modalité et de la tonalité est la source de plusieurs équivoques, elle peut aussi provoquer une discontinuité dans le langage harmonique. En effet, certains passages bien délimités s'inscrivent dans une modalité qui n'a plus rien à voir avec la tonalité : ils relèvent de ce que j'appelle la modalité autonome. Par ailleurs, dans le contexte de cet article, l'appellation séquence harmonique désigne un procédé qui consiste en la transposition répétitive et prévisible d'une progression harmonique donnée (par secondes, par tierces ou par quartes, ascendantes ou descendantes). Parmi les progressions harmoniques les plus souvent employées, celles par tierces se

7Françoise Gervais, Étude comparée des langages harmoniques de Fauré et Debussy, coll. « La Revue musicale ", ñ 272 (Paris : Richard-Massé, 1971); version publiée de sa thèse de doctorat soutenue à La Sorbonne en 1954.

8Edward R. Phillips, « Smoke, Mirrors ans Prisms: Tonal Contradiction in Fauré », Music Analysis 12, n* 1 (mars 1993) : 3-24.

9 Ken Johansen, « Gabriel Faure, un art de l'équivoque », Revue de musicologie 85, n" I (1999) : 63-96. 
démarquent, au point que l'on peut parler d'une harmonie des médiantes, pour reprendre l'expression de Nicolas Meuùs ${ }^{10}$. En raison des enchaînements par tierces avec une seule note commune, l' harmonie des médiantes va de pair avec les fausses relations et avec ce que je nomme le chromatisme des fonctions : bien que Fauré conserve le plus souvent des lignes diatoniques (contrairement aux compositeurs germaniques de son époque), il fait appel à tous les degrés de la gamme chromatique pour la fondamentale de ses accords. Enfin, je nomme contrepoint directionnel une superposition de lignes mélodiques dont la conduite est suffisamment prévisible pour servir de point de repère : séquence mélodique, mouvement conjoint, emploi de motifs et de variantes, imitation, etc.

Compte tenu du fait que mon approche est centrée sur l'écriture et que, par conséquent, elle découle de la tonalité classique, je mettrai d'abord en lumière les éléments qui rattachent l'écriture de Fauré au langage tonal : rôle de la dominante et procédés cadentiels. Puis, je soulignerai les éléments à la frontière de l'ancien et du nouveau : accords substituts, enchaînements équivoques et traitement des dissonances. Ensuite, je décrirai des techniques nettement nouvelles : fonctions harmoniques et procédés d'écriture linéaire. Enfin, je situerai ces techniques dans leur contexte compositionnel : forme et équilibre tonal, esthétique globale.

Cette étude approfondie du langage fauréen s'appuie sur des exemples tirés des 13 Nocturnes pour piano. Ce corpus me semble tout à fait représentatif du style, puisqu'il présente une grande diversité de procédés harmoniques, en plus de couvrir les différentes étapes de la vie du compositeur. Avec les Barcarolles, les Nocturnes représentent une contribution majeure de Fauré au répertoire pianistique. Fauré reprend l'héritage là où l'a laissé Chopin : le nocturne n'est plus une mélodie sentimentale, comme chez Boscha ou Field, mais une œuvre d'une grande profondeur, qui n'évoque pas la nuit mais plutôt le sentiment d'écoute qu'elle éveille. Comme l'affirme Jankélévitch ${ }^{11}$, le nocturne procède d'une esthétique de l'" indistinction ", qui est aussi celle du clair-obscur fauréen. Pour Fauré, le nocturne ne représente pas un genre de peu d'importance. Au contraire, il occupe une place comparable à celle de la sonate chez Beethoven. Si les quatre premiers nocturnes rappellent parfois des couleurs et des formules que l'on retrouve chez Chopin, une originalité incontestable s'affirme dès le Nocturne $n^{\circ} 5$, notamment avec la cadence au ton de la tierce supérieure qui ouvre l'œuvre. Quant au Nocturne $n^{\circ} 6$, il marque une nette évolution dans les procédés harmoniques utilisés, en particulier dans une section centrale aux couleurs très " aériennes ». Enfin, la pensée musicale par lignes, et non plus par accords, prend une importance croissante jusqu' au Nocturne $n^{\circ} 13$. Sans dénaturer son essence, Fauré donne au nocturne une place significative au $\mathrm{XX}^{\mathrm{e}}$ siècle, grâce au renouvellement de l'écriture tonale et pianistique qui s'y opère. Afin de mieux situer dans leur contexte les exemples qui seront présentés, le tableau 1 indique succinctement les principaux faits à retenir pour chacun des Nocturnes.

10Nicolas Meuùs, "À propos du rôle de l'harmonie des médiantes dans l'œuvre de Debussy », dans Mélanges de musicologie 1, dir. par Philippe Mercier et Monique de Smet, Publications d'histoire de l'art et d'archéologie de l'Université catholique de Louvain IV (Louvain : Institut supérieur d'archéologie et d'histoire de l'art, 1974), 27-36; republié dans Fascicules d'Analyse Musicale 4 (1991) : 83-94.

1 I Vladimir Jankélévitch, Le nocturne (Paris : Abin-Michel, 1957). 
Tableau 1. Aperçu d'ensemble des 13 Nocturnes de Fauré ${ }^{12}$

\begin{tabular}{|c|c|c|c|c|}
\hline $\begin{array}{l}\text { Numéro } \\
\text { d'ordre }\end{array}$ & $\begin{array}{l}\text { Numéro } \\
\text { d'opus }\end{array}$ & $\begin{array}{l}\text { Année(s) de } \\
\text { composition }\end{array}$ & Tonalité & $\begin{array}{l}\text { Euvres composées } \\
\text { à la même période* }\end{array}$ \\
\hline $\mathrm{N}^{0} 1$ & Op. $33, n^{0} 1$ & v. 1875 & $M i$ bémol mineur & $\begin{array}{l}\text { Sonate pour violon et piano } \\
n^{\circ} \text { l en la majeur, op. } 13 \\
(1875-76)\end{array}$ \\
\hline $\mathrm{N}^{0} 2$ & Op. $33, n^{\circ} 2$ & v. 1881 & $S i$ majeur & $\begin{array}{l}\text { Quatuor pour piano et cordes } \\
n^{\circ} \text { l en do mineur, op. } 15 \\
(1876-79)\end{array}$ \\
\hline $\mathrm{N}^{0} 3$ & Op. $33, n^{\circ} 3$ & 1883 & La bémol majeur & $\begin{array}{l}\text { Barcarolle } n^{\circ} \text { l en la mineur, } \\
\text { op. } 26(1881 \text { ?) }\end{array}$ \\
\hline $\mathrm{N}^{0} 4$ & Op. 36 & 1884 & $M i$ majeur & $\begin{array}{l}\text { Barcarolle } n^{\circ} 2 \text { en sol majeur, } \\
\text { op. } 41 \text { (1885); } \\
\text { Barcarolle } n^{\circ} 3 \text { en sol bémol } \\
\text { majeur, op. } 42 \text { (1885) }\end{array}$ \\
\hline $\mathrm{N}^{0} 5$ & Op. 37 & 1884 & $S i$ bémol majeur & $\begin{array}{l}\text { "Les roses d'Isaphan ": } n^{\circ} 4 \\
\text { des Chansons, op. } 39 \text { (v. 1884) }\end{array}$ \\
\hline $\mathrm{N}^{\circ} 6$ & Op. 63 & 1894 & $R e ́$ bémol majeur & $\begin{array}{l}\text { Quatuor pour piano et cordes } \\
n^{\circ} 2 \text { en sol mineur, op. } 45 \\
(1885-86)\end{array}$ \\
\hline $\mathrm{N}^{0} 7$ & Op. 74 & 1898 & Do dièse mineur & $\begin{array}{l}\text { Pelléas et Mélisande, } \\
\text { op. } 80 \text { (1898) }\end{array}$ \\
\hline $\mathrm{N}^{0} 8$ & Op. $84, n^{0} 8$ & v. $1869-1902$ & $R e ́$ bémol majeur & $\begin{array}{l}\text { [La révision tardive du } \\
\text { Nocturne } n^{\circ} 8 \text { et l'ajout } \\
\text { postérieur du titre, décidé par } \\
\text { l'éditeur, rend difficilement } \\
\text { justifiable l'établissement } \\
\text { d'une parallèle historique avec } \\
\text { d'autres cuvres.] }\end{array}$ \\
\hline $\mathrm{N}^{\circ} 9$ & Op. 97 & v. 1908 & Si mineur & $\begin{array}{l}\text { La chanson d'Ève, op. } 95 \\
(1906-10)\end{array}$ \\
\hline $\mathrm{N}^{\circ} 10$ & Op. 99 & 1908 & Si mineur & $\begin{array}{l}\text { Préludes pour piano, op. } 103 \\
\text { (1909) }\end{array}$ \\
\hline No 11 & Op. 104, no 1 & 1913 & $\mathrm{Fa}$ dièse mineur & Pénélope (1907-12) \\
\hline $\mathrm{N}^{0} 12$ & Op. 107 & 1915 & Mi mineur & $\begin{array}{l}\text { Barcarolle } n^{0} 12 \text { en mi bémol } \\
\text { majeur, op. } 106 \text { bis (1915) }\end{array}$ \\
\hline$N^{0} 13$ & Op. 119 & 1921 & Si mineur & $\begin{array}{l}\text { Quintette pour piano et cordes } \\
n^{\circ} 2 \text { en do mineur, op. } 115 \\
(1919-21)\end{array}$ \\
\hline
\end{tabular}

* Le parallèle à faire entre les nocturnes et les autres œuvres de Fauré pourrait constituer à lui seul l'objet d'un autre article. Le but du tableau est plutôt de mettre en parallèle le nocturne concerné avec d'autres œuvres significatives de Fauré à peu près contemporaines, simplement à titre de repère historique.

12Les dates qui figurent dans ce tableau sont tirées de Jean-Michel Nectoux, Gabriel Fauré, Les voix du clair-obscur (Paris : Flammarion, 1990). C'est d'ailleurs ce même ouvrage qui a inspiré l'approche générale adoptée ici pour l'étude des Nocturnes de Fauré. 


\section{LE RÔLE DE LA DOMINANTE}

Le mouvement $\mathrm{I}-\mathrm{V}-\mathrm{I}$ constitue le fondement essentiel de la musique tonale ${ }^{13}$. La dominante représente le point culminant de toute progression harmonique et, de ce fait, est intimement liée au processus cadentiel. Chez Fauré, la dominante apparaît toujours avec sa septième, parfois avec sa neuvième. De plus, sa fonction harmonique est plus ou moins claire dans un contexte où l'approche, la résolution, voire l' accord lui-même sont fréquemment modifiés. Avant de parler des dominantes liées aux cadences, précisons qu'en cours de phrase, la fréquence des accords de dominante varie selon l'époque de composition. C'est ainsi que, dans le Nocturne $n^{0} 1$, l'alternance de $\mathrm{V}$ et de I est proportionnellement plus abondante que toute autre progression. Le Nocturne $n^{\circ} 13$, par contre, limite la présence des dominantes à de rares moments cadentiels. Entre ces deux extrêmes, le Nocturne $n^{0} 6$ fait alterner les passages plus tonaux - avec dominantes - et les passages modaux ou non directionnels tonalement.

Il faut aussi mentionner - et c'est là l'un des traits marquants de l'harmonie fauréenne - que de nombreux accords de septième de dominante sont employés de manière non fonctionnelle, c'est-à-dire indépendamment de leur fonction de dominante habituelle en musique tonale. En quelque sorte, l'accord de septième acquiert un statut de consonance, et son utilisation devient comparable à celle des accords parfaits dans un langage plus consonant. Par ailleurs, la distinction entre une septième de dominante fonctionnelle ou non fonctionnelle est liée à son contexte immédiat, c'est-à-dire à son approche et à sa résolution. En musique tonale, préparer une dominante par un deuxième ou un quatrième degré est nettement plus directionnel qu'une préparation par le troisième degré, qui cause une certaine surprise. Or, c'est précisément par ce genre de brouillage que commencent à opérer les subtilités de l'harmonie fauréenne (exemple 1).

Dans l'exemple 1A, en mi bémol majeur, la dominante est non seulement approchée par l'accord du troisième degré, mais celui-ci est de plus abaissé (accord de sol bémol). Cet éloignement tonal lors de l'approche immédiate de

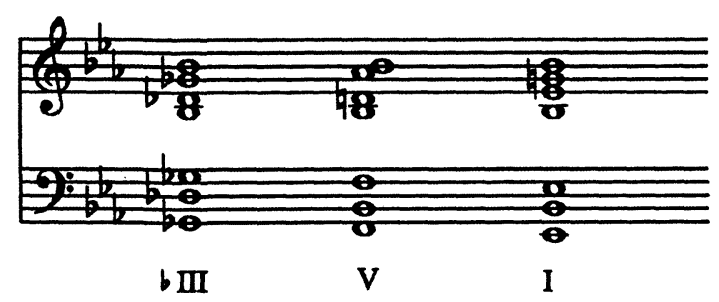

Exemple 1A. Nocturne $n^{0} 4$, mes. 78-84 (réduction)

13 Plusieurs auteurs ont démontré ce fondement, comme Heinrich Schenker, Harmony, dir. et ann. par Oswald Jonas, trad. de l'allemand par Elisabeth Mann Borges (Chicago : University of Chicago Press, 1954); publié à l'origine sous le titre de Harmonielehre (Vienne : Universal, 1906). Voir aussi Richard Franko Goldmann, Harmony in Western Music (New York : Norton, 1965). 


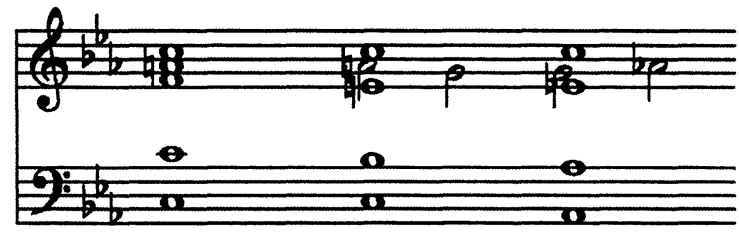

$\begin{array}{cc}\begin{array}{cc}\text { IV } 6 & \text { III 7+ } \\ 4 & \end{array} \\ \text { V/VI (I 6/4 } & \text { V) }\end{array}$

Exemple 1B. Nocturne $n^{0} 3$, mes. 112-20 (réduction)

la cadence crée une coloration très particulière. Par contre, dans l'exemple 1B, en la bémol majeur, ce n'est pas la fondamentale de l'accord du troisième degré qui est altérée, mais sa tierce, $m i$ bécarre. En outre, l'ajout d'une septième et l'approche par sixte et quarte confèrent à ce troisième degré l'apparence trompeuse d'une dominante de $f a(\mathrm{~V} / \mathrm{VI})$, d'où l'équivoque de la résolution sur la tonique du ton principal, la bémol.

Le brouillage contextuel de la dominante peut aussi provenir de l'affaiblissement de la tonique, notamment par l'emploi d'une position de sixte et quarte. Dans la tonalité classique, cette position est instable et appelle nécessairement une résolution. C'est son emploi comme accord autonome, non résolu, qui provoque l'affaiblissement tonal. Toutefois, l' harmonie gagne en coloration et acquiert une mobilité nouvelle, comme l'illustre l'exemple 2.
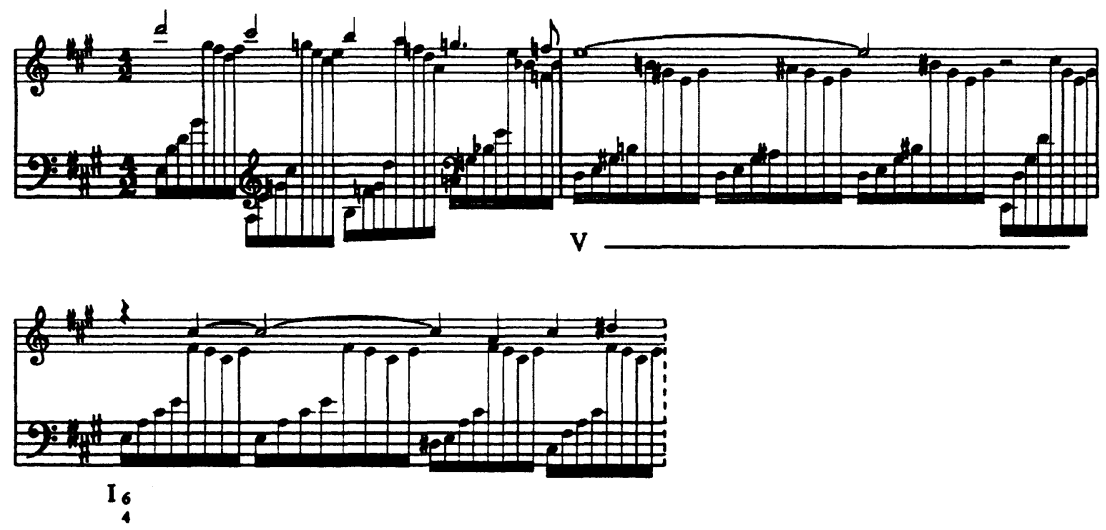

Exemple 2. Nocturne $n^{\circ}$ 6, mes. 70-72

Parfois, la dominante reste suspendue, sans qu'aucune résolution ne se produise. Dans l'exemple 3, l'accord de $f a$ dièse majeur apparaît clairement comme une dominante à la fin de la mesure 15 , mais l'absence de lien immédiat avec ce qui suit correspond à une disparition momentanée du contexte tonal. En effet, l'enchaînement de cette dominante avec l'accord de septième sur do bécarre crée un triton entre les fondamentales, un intervalle exclu du cadre de la tonalité classique. 


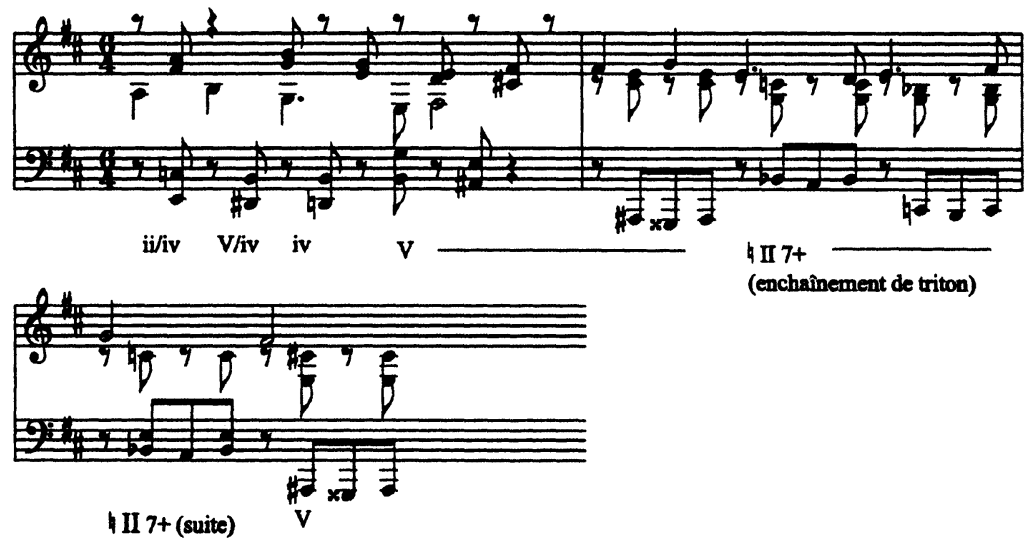

Exemple 3. Nocturne $n^{0}$ 9, mes. 15-17

Malgré le contexte harmonique dissous de l'exemple précédent, on observe que la dominante revient comme pôle harmonique intermittent, bien qu'elle soit privée de toute approche ou résolution usuelle. Cette interpolation d'accords parfois éloignés du ton principal, entre les retours périodiques de la dominante, est un procédé courant à partir du Nocturne $n^{\circ} 6$. Seule la fonction de dominante possède la force nécessaire pour assurer un point de repère suffisamment fort pour maintenir un centre de gravité tonal.

Dans de nombreux passages, cependant, les enchaînements harmoniques demeurent plus près de la tonalité classique qu'il ne le semble, notamment en raison de l'enharmonie; l'équivoque est alors graphique, mais non auditive. Dans l'exemple 4, la réécriture des accords selon leur véritable « orthographe harmonique " permet de ramener la progression à des enchaînements plutôt usuels pour l'époque. Par contre, ce sont les lignes mélodiques qui altèrent la

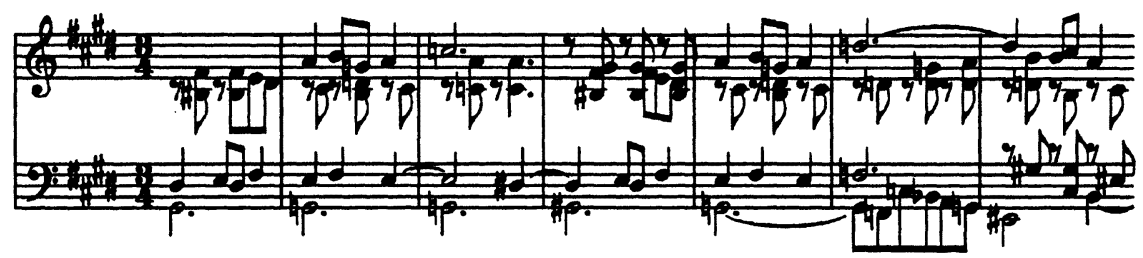

Reteriture harmonique

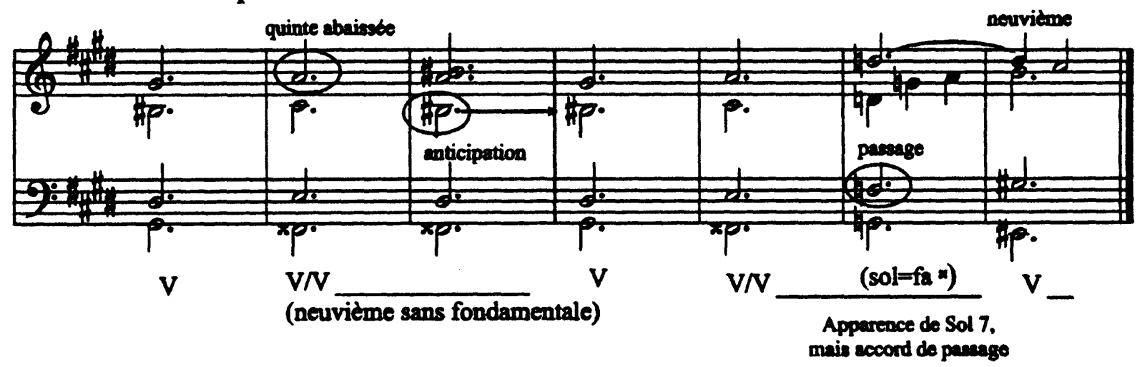

Exemple 4. Nocturne $n^{\circ} 6$, mes. 37-43, accompagné d'une réécriture harmonique 
perception claire de l'accord. Le do bécarre de la mesure 39 n'est que l'anticipation de la sensible de $\mathrm{V}$, mais l'accentuation que lui confère sa doublure et le mouvement disjoint du soprano amenuisent le contraste entre les deux accords, d'où un brouillage dans l'enchaînement.

Le jeu combiné des altérations chromatiques - notamment l'abaissement de la quinte - et des notes étrangères peut donc modifier substantiellement la sonorité d'un accord de dominante, au point de le rendre difficilement perceptible. Bien plus, les rencontres de notes ainsi produites peuvent à leur tour susciter la confusion en générant des accords ornementaux. Dans l'exemple 4, l'accord de la mesure 42, qui est pourtant simple à analyser lorsqu'on comprend la conduite des voix sous-jacente, peut s'entendre momentanément comme un accord de sol. L'auditeur éprouve donc une tension entre l'accord qu'il entend momentanément et le contexte harmonique global, qui n'est confirmé qu'à certains moments du discours.

Enfin, l'absence prolongée de la dominante et de la sensible du ton principal contribue à rendre équivoque la tonalité d'un passage. Au début du Nocturne $n^{\circ} 4$ (exemple 5), la dominante de mi bémol majeur n'apparaît pas avant la dixième mesure. En outre, aucun des degrés forts de la tonalité n'est entendu avant ce moment. Par contre, un mécanisme compensatoire de cycle des quintes (V/vi, V/ii, V/V, V), à la fin de la phrase, rétablit avec clarté la hiérarchie tonale ${ }^{14}$.
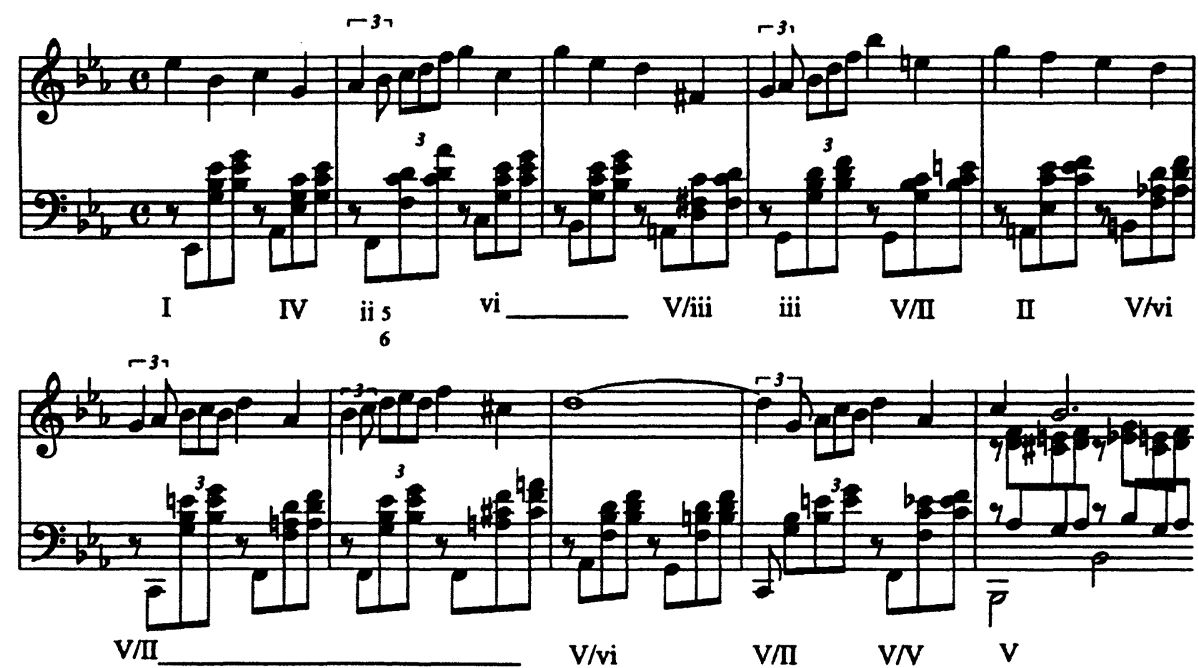

Exemple 5. Nocturne ${ }^{0}$ 4, mes. 1-10

14Le cycle de quintes constitue le procédé d'affirmation tonale usuel dans le langage harmonique classique. 
Dans les derniers nocturnes, Fauré va encore plus loin en éliminant pendant de longs moments tout accord de dominante, même non fonctionnel, comme dans l'exemple 14 (voir plus loin).

\section{LES PROCÉDÉS CADENTIELS PARTICULIERS}

En plus de brouiller l'environnement immédiat de la dominante, le langage fauréen induit, à plus grande échelle, des zones de prédominantes qui s'écartent du langage tonal, suscitant ainsi des couleurs cadentielles nouvelles. Un premier processus consiste à établir de justesse une tonalité éloignée à la fin de la phrase. Dans l'exemple 6, ce sont les transformations successives d'un accord qui permettent ce revirement. Un accord de dominante, sur la, est d'abord déstabilisé par l'arrivée de la neuvième, si bémol, à la basse. Ensuite, la note $m i$, quinte de l' accord, est abaissée. Par enharmonie de la sixte allemande ainsi obtenue avec la septième de dominante, l'accord est réinterprété comme une septième sur $m i$ bémol. Le mouvement caractéristique d'un cycle de quintes, à la basse, permet d'introduire la nouvelle tonique de manière claire.
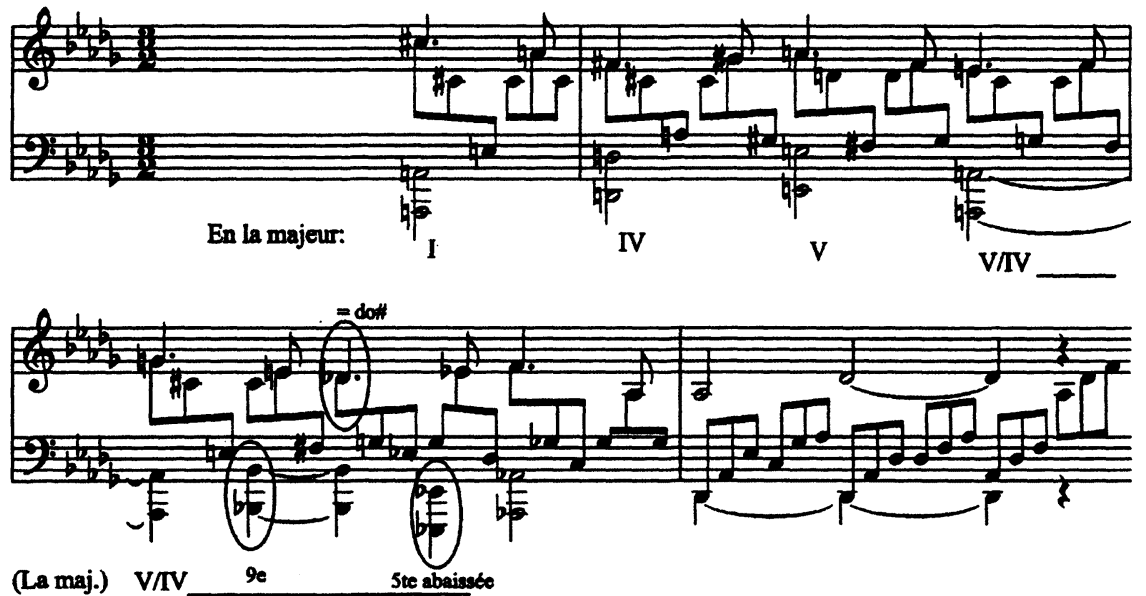

En ré bermol majeur

$\mathbf{V} / \mathbf{V}$ V

I

Exemple 6. Nocturne $n^{0}$ 6, mes. 6-9

Cette bifurcation surprenante n'empêche pas la cadence de demeurer claire ni traditionnelle par le rôle structurant réservé à la dominante. Dans les derniers nocturnes, toutefois, une modification plus fondamentale s'opère : la dominante demeure présente, mais plus estompée, et accompagnée de la sous-dominante au sein même du processus cadentiel. C'est comme si, du camouflage de plus en plus marqué des fonctions tonales, résultait la nécessité de cadences présentant une sonorité moins classique. Soit ces sonorités cadentielles sont librement empruntées aux cadences modales et diatoniques du plain-chant ${ }^{15}$, 
comme la cadence dite « à la Fauré » (exemple 7), soit elles font appel à l'harmonie des médiantes (exemple 8).

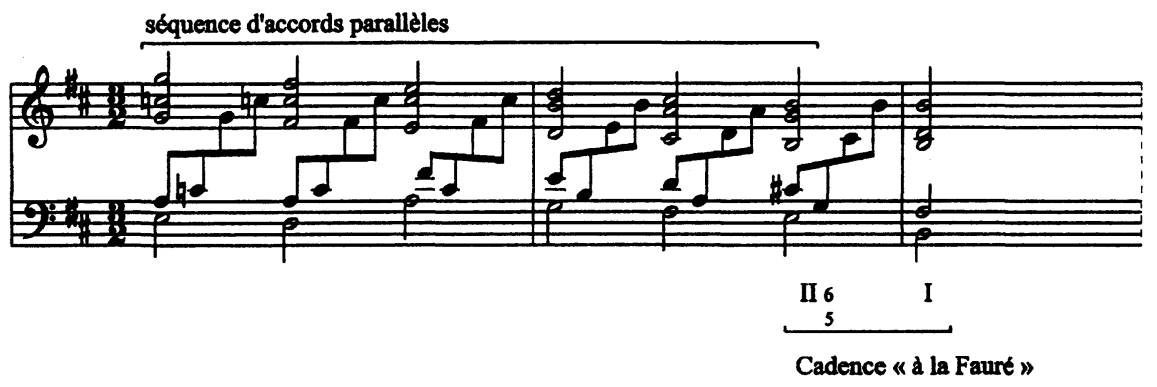

Exemple 7. Nocturne $n^{\circ} 13$, mes. 146-48 (réduction)
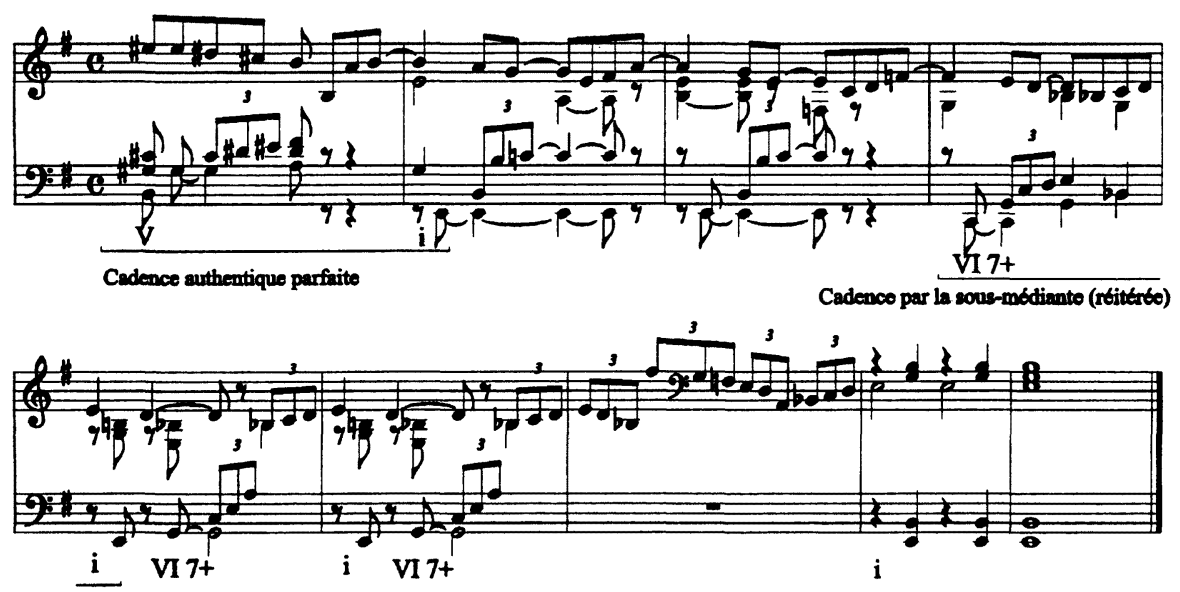

Exemple 8. Nocturne $n^{\circ}$ 10, mes. 67-75

Dans l'exemple 7, il est intéressant d'observer le contexte où se produit la cadence. La succession d'accords de septième par mouvement parallèle crée une couleur non tonale, presque debussyste par son parallélisme ${ }^{16}$. Néanmoins, il faut garder à l'esprit que les cadences modales ne sont jamais employées en remplacement de la dominante, mais bien en complément de celle-ci, comme réitération modale d'une cadence d'abord affirmée tonalement. Il ne serait donc pas exact d'affirmer, comme le fait Johansen, que Fauré invente des couleurs nouvelles en employant d'autres fonctions que la dominante pour cadencer ${ }^{17}$.

for Gabriel Fauré » (thèse de doctorat, University of Chicago, 1973).

16Cette couleur, particulière à Fauré, est un élément stylistique propre. Les progressions de septièmes parallèles de la fin du romantisme faisaient plutôt usage de septièmes de dominante ou de septièmes diminuées, notamment chez Liszt.

17Johansen, « Gabriel Fauré, un art de l'équivoque », 75. 
Pour appuyer son affirmation, Johansen renvoie au début du Nocturne $n^{\circ} 9$ (cadences sur V, VII, V/V et $\mathrm{i}$, selon Johansen), reproduit à l'exemple 9.

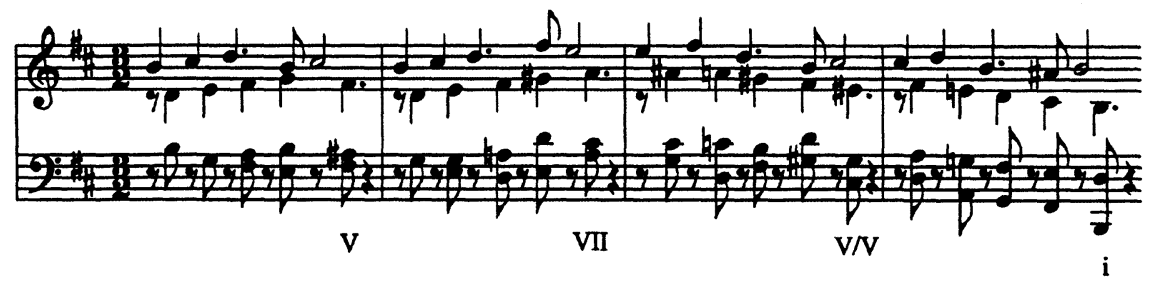

Exemple 9. Nocturne $n^{\circ}$ 9, mes. 1-4

Je remets en cause le sens donné au mot " cadence " par Johansen. Il est important d'établir une distinction entre une ponctuation rythmique du discours et une cadence, liée à un processus harmonique directionnel orienté vers la dominante ${ }^{18}$. Or, le début du Nocturne $n^{\circ} 9$ me semble un bon exemple de ponctuations répétées, mais non cadentielles en raison de leur fréquence (à toutes les mesures) et de leur rôle très localisé. Des exemples plus vraisemblables de cadences apparemment non tonales en raison de leur degré d'appui autre que I ou V - se retrouvent plutôt dans le Nocturne $n^{\circ} 5$ (exemple 10). Dans ce cas, la cadence se produit sur le troisième degré avec une tierce majeure, étrangère au ton initial. C'est un procédé qui n'est pas propre à Fauré, et que l'on retrouve notamment chez Brahms ${ }^{19}$. Le troisième degré joue alors un rôle de substitut de V. En musique tonale, la notion d'accord substitut concerne $\mathrm{V}$ et $\mathrm{I}$. Substituer à l'accord attendu un accord qui lui ressemble demande un degré de prévisibilité que seules la dominante et la tonique peuvent assurer. Ajoutons que, dans le contexte de l'évolution du langage fauréen, l'emploi cadentiel du troisième degré traduit l'importance croissante de l'harmonie des médiantes.

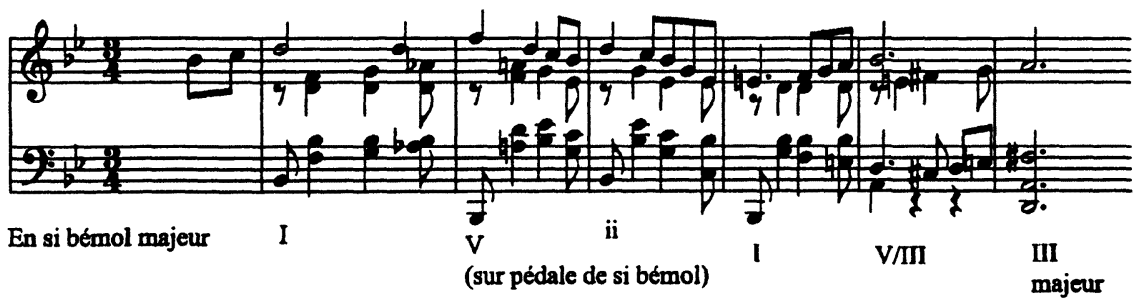

Exemple 10. Nocturne $n^{\circ} 5$, mes. 1-6 (réduction)

18C'est aussi le sens du mot « cadence » lorsqu'il est employé par la plupart des théoriciens, comme Schoenberg et Rosen. Voir Arnold Schoenberg, Structural Functions of Harmony (Londres : Williams and Norgate, 1954); Charles Rosen, "The Coherence of the Musical Langage », dans The Classical Style: Haydn, Mozart, Beethoven, édition augmentée (New York et Londres : Schirmer, 1997), 57-98.

19 Par exemple, la Rhapsodie en sol mineur, op. 79, n" 2, de Johannes Brahms, comporte une première cadence sur sol majeur, à la mesure 4, puis une deuxième sur si majeur, à la mesure 8. L'arrêt sur ces deux accords met en évidence leur relation de tierce. 


\section{ACCORD SUBSTITUT ET POLARISATION SECONDAIRE}

Encore en écho à l'article de Ken Johansen, il me semble nécessaire d'apporter quelques précisions sur les équivoques tonales que l'on retrouve chez Fauré. Tout d'abord, Johansen affirme que :

[Les] expériences musicales passées nous ont conditionnés à éprouver certaines attentes fondées sur des événements et des suites d'événements musicaux devenus, par répétition, des conventions idiomatiques. [...] Si nous sommes étonnés à l'audition d'une cadence rompue (bien que nous ne le soyons peut-être plus), c'est parce que nous attendions une cadence parfaite ${ }^{20}$.

Le couple dominante-tonique vient certes préciser en partie les attentes tonales de l'auditeur, mais il est tout de même souhaitable d'élargir quelque peu ces attentes par la notion d'accord substitut, qui a été introduite dans le commentaire de l'exemple 10. Cette notion s'applique non seulement à un contexte cadentiel, mais aussi à toute progression en cours de phrase. La comparaison des harmonies suggérées par la ligne de basse et celles employées par Fauré, au début du Nocturne $n^{\circ} 6$, permettra d'en comprendre le fonctionnement (exemples 11 et 12).

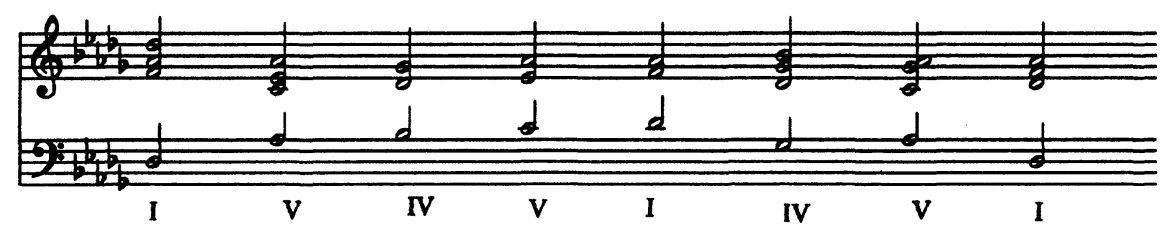

Exemple 11. Nocturne $n^{\circ}$ 6, mes. 1-3 : harmonie suggérée par la basse (réduction)
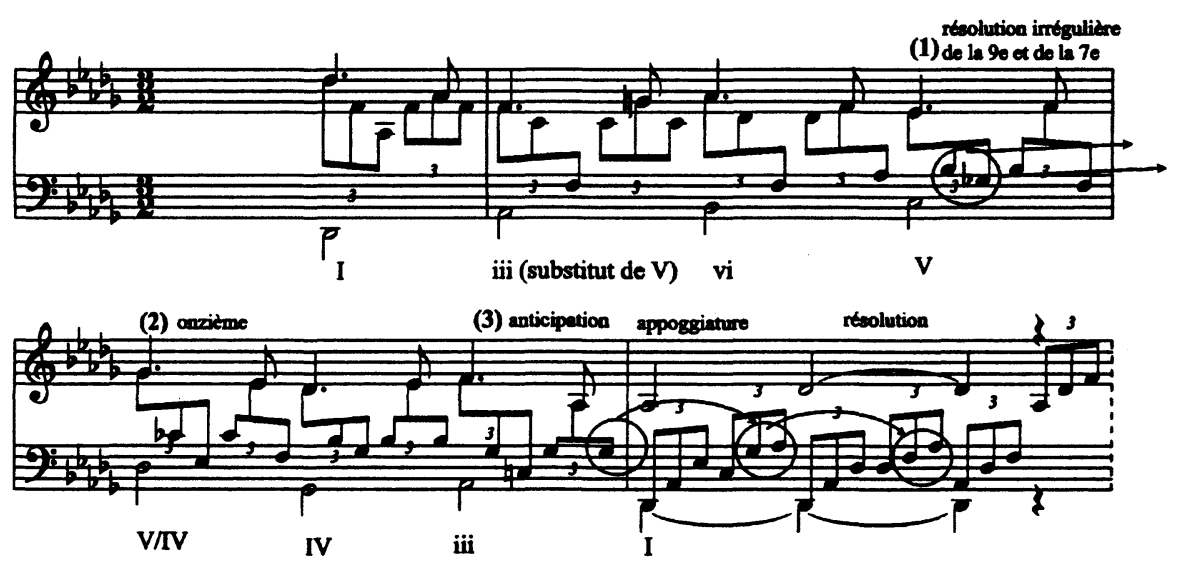

Exemple 12. Nocturne $n^{\circ}$ 6, mes. 1-3 : harmonie employée 
La basse, limpide sur le plan tonal, suggère une harmonisation assez simple. Or, le troisième degré est employé comme substitut de V. De plus, il est intéressant de constater que les accords de tonique (sauf le premier) et de dominante sont systématiquement brouillés : mouvements irréguliers de la septième et de la neuvième sur la dominante du ton principal (1); accord de onzième attaqué par mouvement direct sur le V/IV (2); et anticipation de l'appoggiature suggérant la septième de dominante sur III (3). Tous ces procédés rendent équivoques les accords de tonique et de dominante, bien que le contexte laisse peu de doute sur l'accord qui est remplacé.

Par ailleurs, lorsque diverses interprétations possibles entrent en conflit, Johansen semble ignorer le concept de polarisation secondaire ${ }^{21}$. Celui-ci $s$ 'avère particulièrement utile pour distinguer les attentes locales de celles qui découlent d'un contexte plus vaste. Par exemple, le début du Nocturne $n^{o} 3$, qui commence en la bémol majeur, fait entendre dès la deuxième mesure une polarisation locale avec la dominante de do mineur (exemple 13). Or, non seulement cette dominante conduit à un substitut de do ( $a, \mathrm{IV}$ en $\mathrm{do}$ ), mais elle est précédée et suivie de l'accord de quinte augmentée sur la tonique, accord par nature équivoque en raison de ses trois possibilités enharmoniques. Enfin, l'accord de ré bémol n'a pas de véritable fonction (accord ornemental), en raison de sa position de sixte et quarte, qui le prive de force harmonique. En outre, le rythme harmonique assez rapide accentue la difficulté pour l'oreille de créer des associations logiques entre les accords. Malgré tout, la tonalité de la bémol majeur demeure solidement en place grâce à la récurrence de la tonique à la basse et de la médiante à la voix supérieure. C'est cette tension conflictuelle entre un accord de tonique réitéré et une polarisation secondaire qui crée l'équivoque.

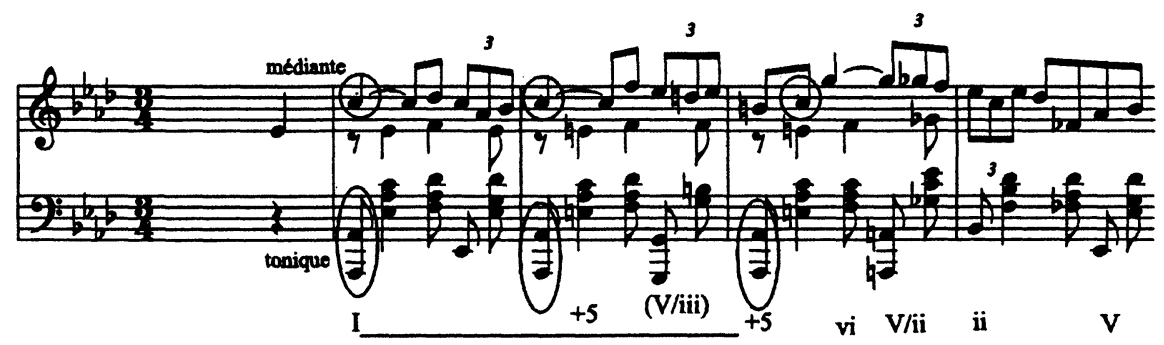

Exemple 13. Nocturne $n^{0}$ 3, mes. 1-4

L'équivoque n'est toutefois pas nécessairement liée à la rapidité du rythme harmonique. Dans le Nocturne $n^{\circ} 9$, une phrase complète est formée d'un accord de quinte augmentée à la fonction indéterminée qui se transforme lentement en dominante (exemple 14). Dans de tels passages, où un accord se transforme lentement avant de se résoudre, le mouvement harmonique demeure suspendu, en ce sens qu'il n'y a plus de progression d'accords mais plutôt une longue métamorphose, ce qui supprime presque l'attente tonale 22 .

22 Dans son article, Johansen affirme que ce passage peut être compris en entier comme une vaste 

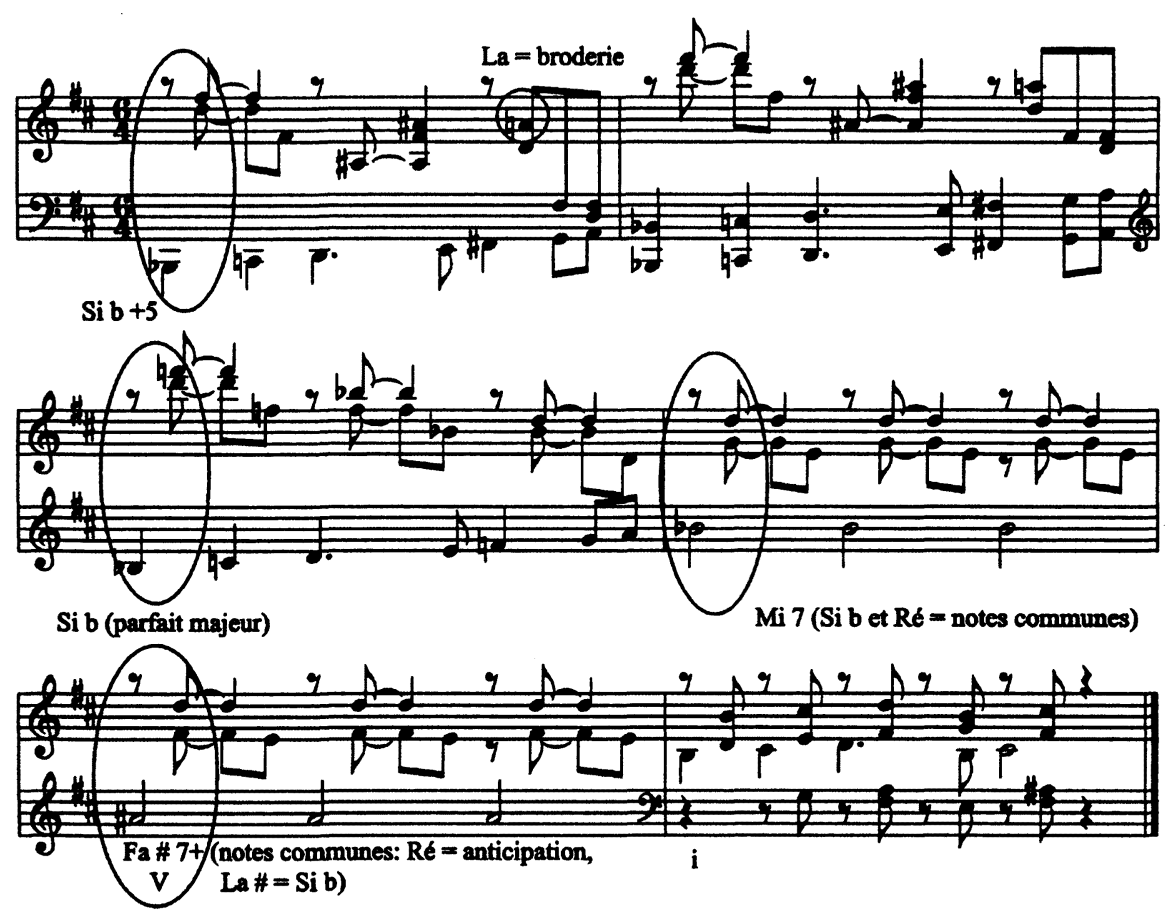

Exemple 14. Nocturne $n^{\circ} 9$, mes. 5-10

L'équivoque peut donc devenir suffisamment déstabilisante pour ne plus se définir comme un jeu d'attentes et de résolutions-surprises. Dans ce cas, elle est davantage liée à la suspension du temps qu'à une progression. D'ailleurs, le caractère moins directionnel de l'écriture modale est l'un des moyens employés par Fauré pour maintenir la suspension. La modalité acquiert ainsi une certaine indépendance dans la durée par rapport la tonalité.

\section{LA MODALITÉ AUTONOME}

Afin de donner à la modalité une certaine autonomie par rapport à la tonalité, Fauré fait cohabiter de manière successive et bien délimitée les deux langages, comme l'illustre la section centrale du Nocturne $n^{o} 6$. Ici, la délimitation est d'abord marquée par une cadence forte, en ré bémol majeur, qui conclut une zone clairement tonale. Puis commence la zone modale, où un quatrième degré haussé donne immédiatement à l'harmonie une coloration lydienne. La succession des accords se fait par tierces - ré bémol (enharmonique à do dièse), la, puis do naturel - ce qui contribue à éviter toute connotation tonale. Ensuite, la commence à s'imposer comme tonique, tout d'abord par son 
rôle de note sommet, à la mesure 69 (exemple 2), puis par un point cadentiel sur un accord de $m i$ avec septième à la mesure 71. L'effet quasi tonal de cette cadence à la dominante, mi, est atténué par une ligne supérieure qui descend en gamme par tons (mes. 70) et par un premier degré peu affirmé sur le plan tonal en raison de sa position en quarte et sixte.

La zone modale se poursuit ensuite, puis est interrompue une première fois par un passage où elle s'oppose, par alternance, à des zones tonales d'une mesure (exemple 15, mes. 80-86). Ici, le lien qui unit les zones tonales entre elles est solidement structuré : la dominante de do dièse est présente aux mesures 80 et 82 , puis se résout à 84 sur le substitut de I, V/IV. D'un point de vue formel, ces insertions tonales proviennent d'une variante de la mesure 37. Aussi, est-il prévisible que la suite du Nocturne (mes. 88-99) répète plus ou moins intégralement les mesures 46 à 56.
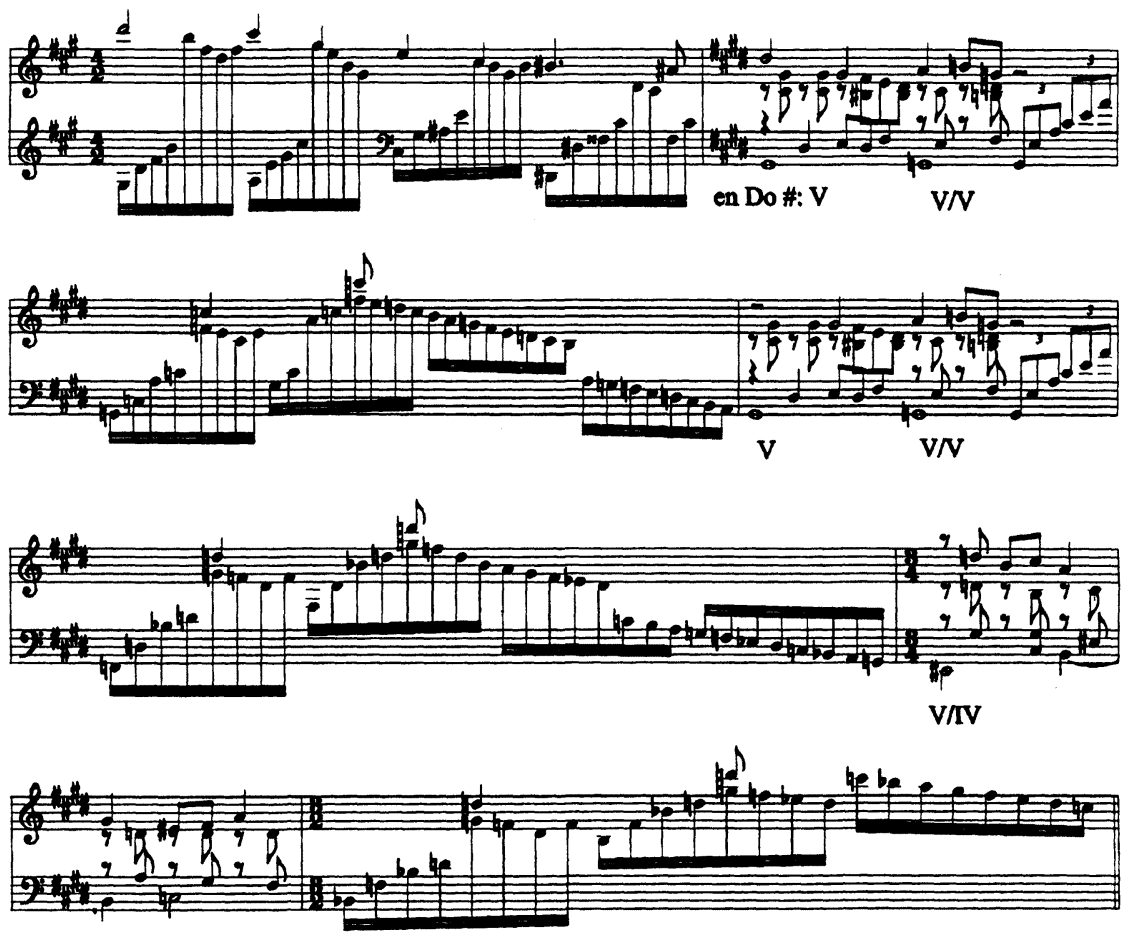

Exemple 15. Nocturne $n^{o}$ 6, mes. 79-86

Un ultime retour de la couleur modale, à la mesure 100 , aboutit à une série de séquences (mes. 106-11). Celles-ci, par récurrence d'un modèle, créent un mouvement directionnel propice à une progression qui ramène la couleur tonale. Ce retour coïncide d'ailleurs avec la réapparition du thème initial, qui devient la conclusion du Nocturne $n^{0} 6$. 


\section{EMPLOI DES DISSONANCES}

D'un point de vue contrapuntique, l'emploi des dissonances chez Fauré demeure relativement classique. Celles-ci ne sont habituellement pas cristallisées en notes ajoutées non résolues, comme chez Debussy. Qu'elles soient parties intégrantes d'un accord dissonant - comme les septièmes et les neuvièmes ou présentes comme notes étrangères - notes de passage, broderies, retards, appoggiatures - leur traitement demeure le plus souvent conjoint, bien que les septièmes et neuvièmes puissent se résoudre en montant dans les voix internes. Or, l'abondance de notes étrangères et de dissonances à toutes les voix, avec leur mouvement conjoint obligé, contribue à rendre la texture fortement contrapuntique. De plus, ces procédés créent une couleur harmonique où les dissonances sont omniprésentes. Les accords de septième constituent la couleur de base du tissu sonore, alors que les accords parfaits sont plus rares. Les rencontres de notes étrangères sont tellement nombreuses que les accords de septième finissent par être perçus comme relativement consonants.

En fait, le degré de dissonance des notes étrangères est lié à la période de composition du nocturne. D'un emploi relativement conventionnel dans les six premiers nocturnes, les notes étrangères prennent plus d'importance dans le Nocturne $n^{o} 7$, plus particulièrement avec l'appoggiature et la syncope, dans un contexte motivique. Toutefois, c'est à partir du Nocturne $n^{\circ} 9$ que l'écriture verticale prend le pas sur une écriture plus nettement par accords. Il devient souvent difficile de percevoir les accords sous-jacents à la conduite des voix bien que ceux-ci demeurent présents. Un accord ne se précise souvent qu'au dernier moment, pour ensuite s'enchaîner sur un nouvel accord dont la construction est elle aussi obscurcie par la multiplication des notes étrangères. Il devient alors important de distinguer ce qui est ornemental de ce qui est fonctionnel. D'une part, une résolution se perçoit avec l'arrivée d'un accord classé qui, même avec une septième, représente un point de stabilité. D'autre part, le contexte fournit une clé d'analyse indispensable, selon qu'il y a récurrence d'un accord, d'une harmonie de dominante ou de tierce, ou encore d'une séquence harmonique.

Il a déjà été question de l'ornementation mélodique souvent abondante des dominantes. Parfois, ce ne sont plus seulement les lignes mélodiques, mais les accords eux-mêmes qui deviennent les appoggiatures d'une résolution. Distinguer la fonction réelle des accords ornementaux demande alors une écoute très subtile, qui n'est pas seulement orientée vers les conventions tonales. Comme on le sait, la notion d' accord en appoggiature sera reprise chez Debussy et aussi, sous une forme plus large, chez Messiaen, dans le cadre du principe «d'agrandissement des notes étrangères ${ }^{23}$ ». Si les enchaînements de l'exemple 16 demeurent difficilement explicables dans un cadre tonal, ils deviennent simples lorsqu'on les entend dans un contexte d'appoggiatures et d'harmonie des médiantes. Pour ce faire, il faut d'abord reconnaître que les accords d'arrivée sont perçus comme tels en raison de leur caractère d'aboutissement : placés en fin de mesure, ils concluent un geste harmonique avant de conduire à une 
nouvelle couleur. D'un point de vue mélodique, les arpèges de mi bémol (mes. 14) et de sol (mes. 16) confiés au soprano ne sont complétés qu'en fin de mesure. Enfin, les accords parfaits qui concluent ces deux mesures constituent un appui non équivoque, du moins à l'écoute. Deux subtilités sont à souligner : les accords des mesures 14 et 16 sont approchés à la tierce par des accords non fonctionnels (do vers $m i$ bémol, $m i$ vers sol), pendant que la basse fait l'appoggiature de la septième de l'accord. Ce sont ces dissonances particulièrement déstabilisantes à l'audition qui rendent le passage équivoque.

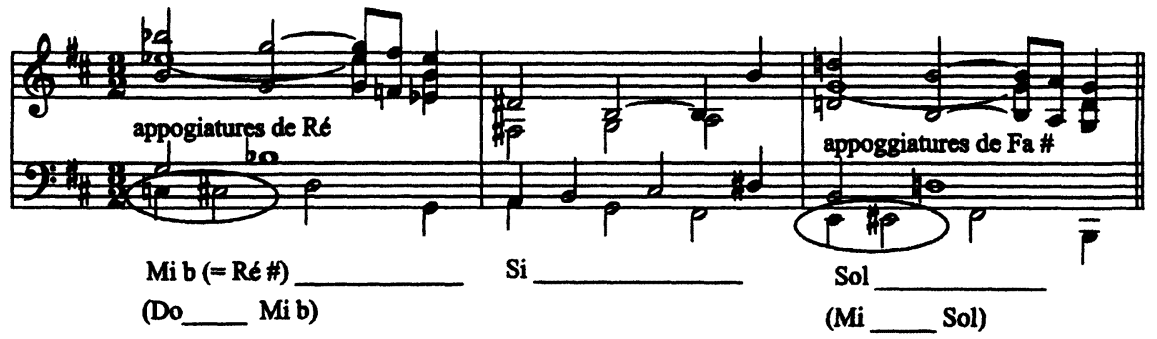

Exemple 16. Nocturne no 13, mes. 14-16

L'exemple ultime d'un contrepoint qui prédomine sur l'harmonie se trouve dans le Nocturne $n^{\circ} 13$ (exemple 17). La première phrase commence par un accord de si mineur. Mais la suite brouille la perception claire des accords, notamment en raison du tuilage des voix, qui sont décalées rythmiquement les unes par rapport aux autres. Certes, des accords identifiables apparaissent momentanément, mais la progression s'explique essentiellement en fonction des lignes mélodiques, et non de la succession des accords. L'harmonie trouve son sens par les lignes descendantes, vers $m i$, que font entendre les deux voix inférieures. Dans de tels passages, Fauré renoue avec un principe d'écriture de la Renaissance en considérant les accords non comme des entités propres, mais plutôt comme la résultante verticale de la conduite des voix.

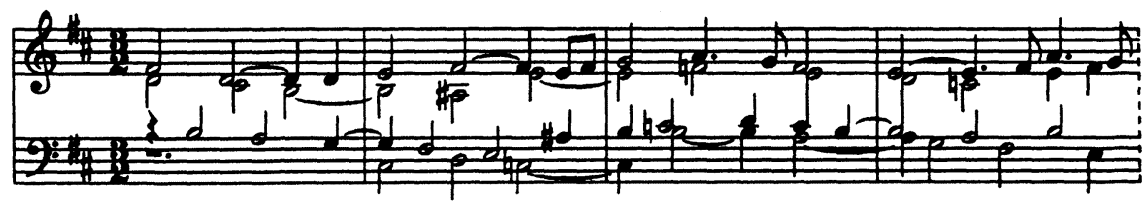

(ligne octavice

d la basse)

Exemple 17. Nocturne $n^{\circ} 13$, mes. 1-4

Enfin, à la notion de dissonances simultanées, on pourrait opposer celle de dissonances successives, lorsque les accords qui se succèdent établissent des contrastes entre eux. Les fausses relations chromatiques sont fréquentes dans les enchaînements à la tierce. Par exemple, la section centrale du Nocturne $n^{\circ} 5$ (exemple 18) oppose l'accord de si bémol mineur à celui de ré bémol mineur. 
Le $f a$ bémol de ce dernier accord, par opposition au $f a$ bécarre du premier, crée un degré de contraste suffisant pour provoquer un rapport dissonant entre les deux accords. Comme l'affirme Ulehla, l'emploi des différents degrés de la gamme chromatique comme moyen de dissonance par succession d'accords s'inscrit dans les principales nouveautés du langage de l'époque fauréenne ${ }^{24}$.

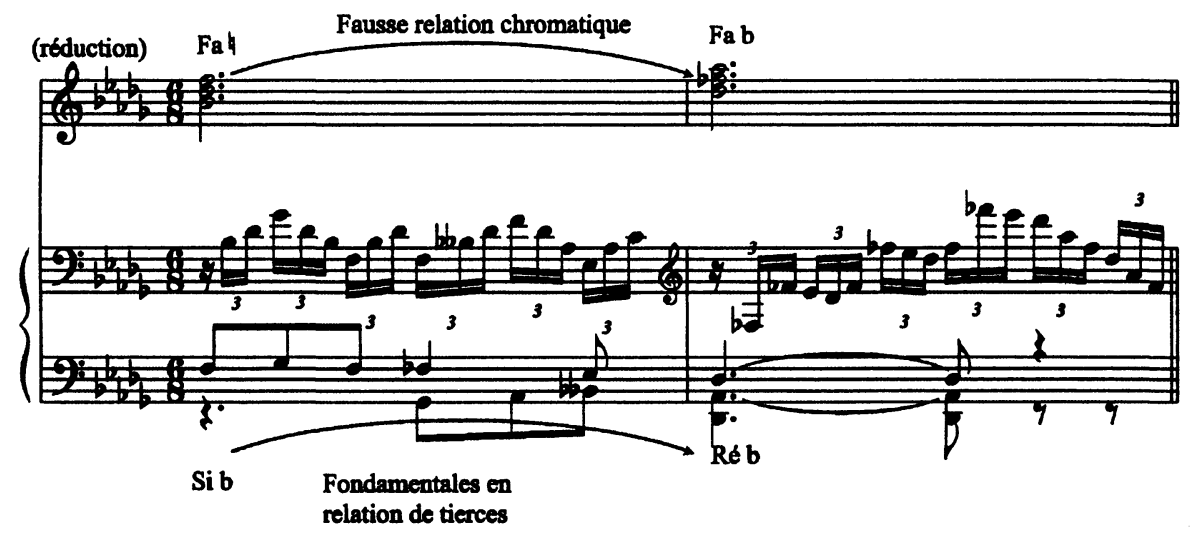

Exemple 18. Nocturne $n^{\circ} 5$, mes. 70-71, accompagné d'une réduction

\section{DE NOUVELLES FONCTIONS HARMONIQUES}

Sous la rubrique des progressions non fonctionnelles, $j$ 'intègre toutes les progressions qui échappent à la tonalité classique. J'ai déjà évoqué l'emploi courant des accords de septièmes de dominante « défonctionnalisés ». Mais la définition du langage ne saurait se limiter à la description de ce qu'il brise par rapport à la norme. Aussi est-il nécessaire de préciser comment Fauré développe de nouvelles fonctions tonales à l'intérieur de son langage harmonique. La question de la fonction, au sens large, est liée aux points de repère qui assurent une stabilité harmonique. Chez Fauré, les nouvelles fonctions s'articulent autour des procédés de séquence harmonique, avec des enchaînements monointervalliques par secondes, par tierces et par tritons. La séquence établit une prévisibilité par la récurrence du modèle dans les reproductions. Elle peut revêtir des formes multiples, des plus simples (séquence d'accords parallèles, exemple 7) aux plus complexes (exemple 19). La séquence était abondamment utilisée dans la tonalité classique. Chez la plupart des compositeurs, dont Bach, elle avait souvent pour rôle d'affirmer la tonalité25. Chez Fauré, elle conserve son rôle structurant, mais elle s'écarte par contre des cadres de la tonalité classique, puisque les accords employés ne se rattachent souvent pas à des fonctions tonales.

Dans l'exemple 19, la reprise à chaque mesure des mêmes motifs à toutes les voix assure la prévisibilité contrapuntique. En même temps, la basse fait 

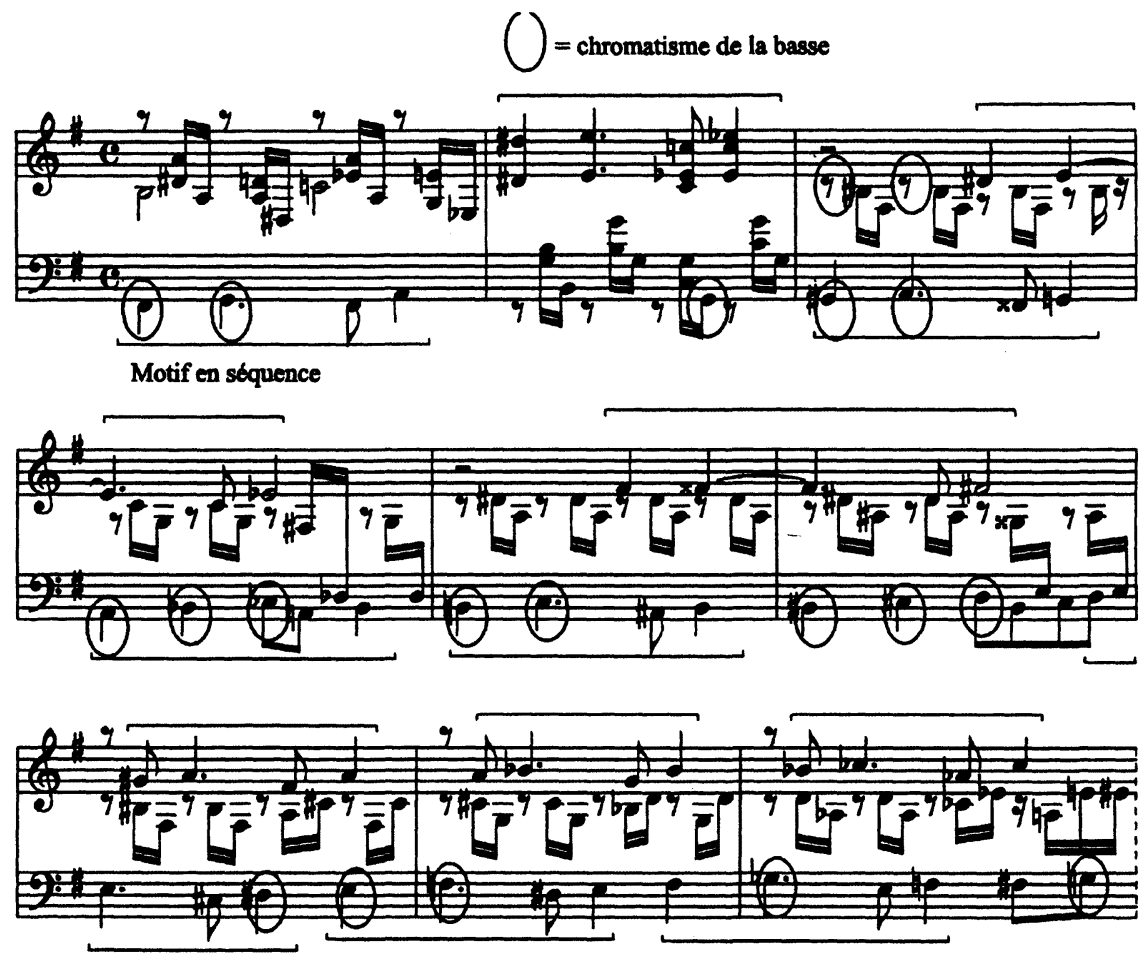

Exemple 19. Nocturne $n^{\circ} 10$, mes. 21-29

entendre une gamme chromatique ascendante, du sol dièse contre grave au sol bécarre grave. Par ailleurs, deux éléments viennent perturber la prévisibilité de la progression : le contrepoint complexe, avec les voix extrêmes en imitation canonique, et le décalage rythmique d'un temps à la basse à partir de la mesure 27, qui modifie sensiblement les appuis rythmiques du motif. Harmoniquement, il y a une progression intervallique ascendante, d'abord en tierces, puis en secondes mineures. Il n'est certes pas impossible d'analyser ce passage selon les règles de la tonalité classique, comme l'a fait Johansen ${ }^{26}$. Mais une telle analyse semble inutilement complexe. En effet, en admettant que Fauré renouvelle la notion même de fonction harmonique, on peut abandonner l'analyse tonale stricte au profit d'une vision par progression intervallique : le passage s'explique alors tout simplement par une oscillation en tierces, suivie d'une montée monointervallique en secondes mineures (exemple 20).

Si les progressions monointervalliques sont souvent associées aux séquences, elles peuvent aussi être employées de manière autonome. Bien entendu, les progressions par quinte, par tierces et par secondes ne sont pas propres à Fauré. Ainsi faut-il préciser en quoi Fauré les emploie de manière originale. Tout d'abord, les enchaînements par quintes, fortement associés à la tonalité 


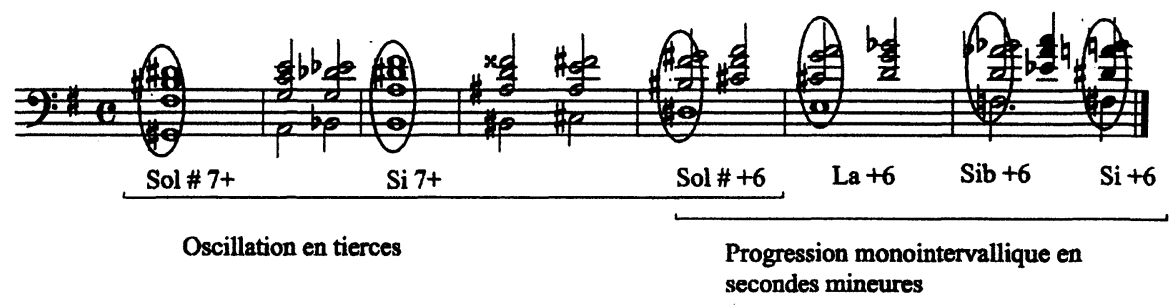

Exemple 20. Nocturne $n^{\circ} 10$, mes. 23-29 (réduction et analyse)

classique, conservent un rôle relativement traditionnel d'affirmation tonale menant à une cadence. Leur originalité, dans le langage fauréen, provient surtout du contexte : ils émergent à la fin d'une section à la tonalité instable, comme mécanisme compensatoire ayant pour but de rétablir le sens tonal.

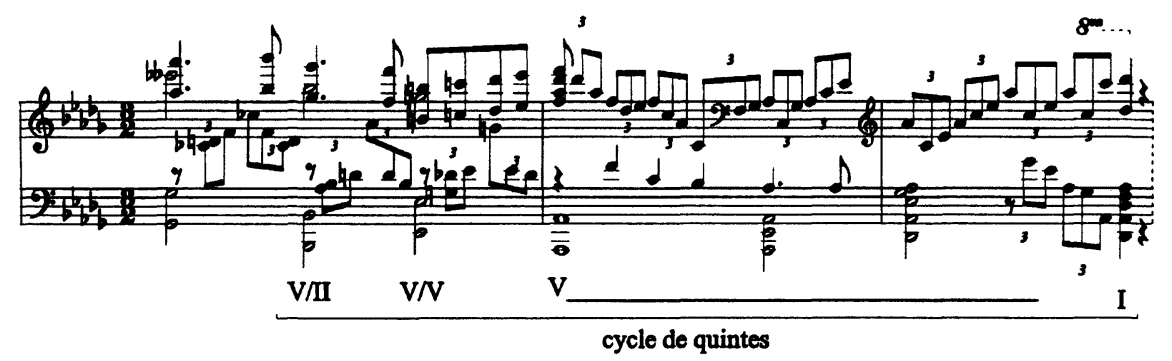

Exemple 21. Nocturne $n^{\circ}$ 6, mes. 16-18

Plus novateurs, les enchaînements par secondes - habituellement majeures agissent également comme progression harmoniquement prévisible. Ces enchaînements peuvent se réaliser avec une conduite des voix non séquentielle lorsqu'il n'y a pas de symétrie mélodique (exemple 22), séquentielle lorsqu'il y a une symétrie mélodique à partir d'un modèle (exemple 22) ou, surtout dans les derniers nocturnes, en accords parallèles, c'est-à-dire lorsque toutes les voix décrivent un mouvement parallèle (exemple 7). En outre, comme dans l'exemple 23, les enchaînements par secondes peuvent s'inscrire dans un contexte de gamme par ton ${ }^{27}$.

La contribution la plus importante de Fauré à l'évolution du langage tonal demeure toutefois l'emploi omniprésent d'enchaînements par tierces, au point que l'on peut véritablement parler d'harmonie des médiantes. Certes, comme l'affirme Nicolas Meuùs, Fauré ne va pas aussi loin que le fera Debussy en ne donnant aucun rôle cadentiel à l'harmonie des médiantes ${ }^{28}$. Sans être nouvelle en elle-même, l'harmonie des médiantes prend toutefois des proportions et des

27Françoise Gervais (voir note 7) avait déjà relevé l'importance de la gamme par tons chez Fauré. Toutefois, je trouve important de préciser que la gamme par ton est souvent employée dans un contexte séquentiel.

28 Meuùs, « À propos du rôle de l'harmonie des médiantes dans l'œuvre de Debussy », 27-36. 


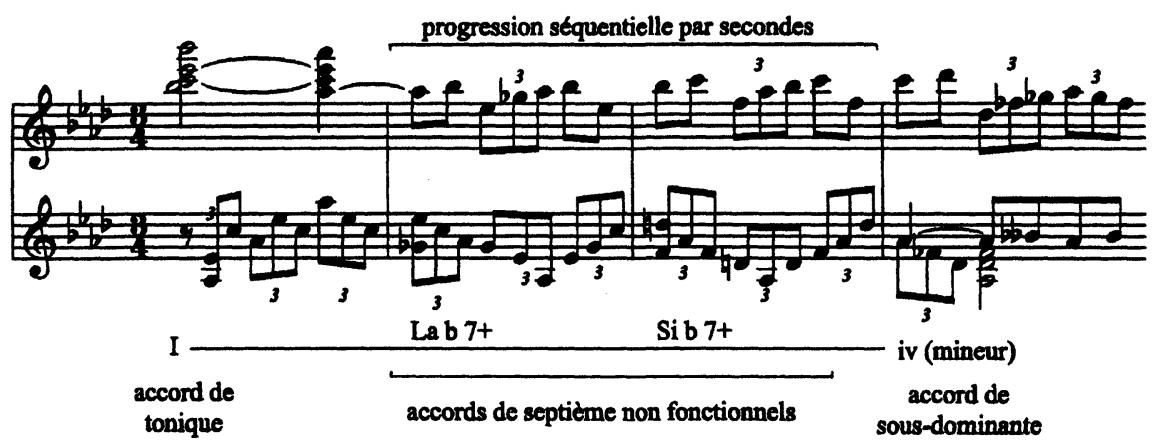

Exemple 22. Nocturne no 3, mes. 101-4

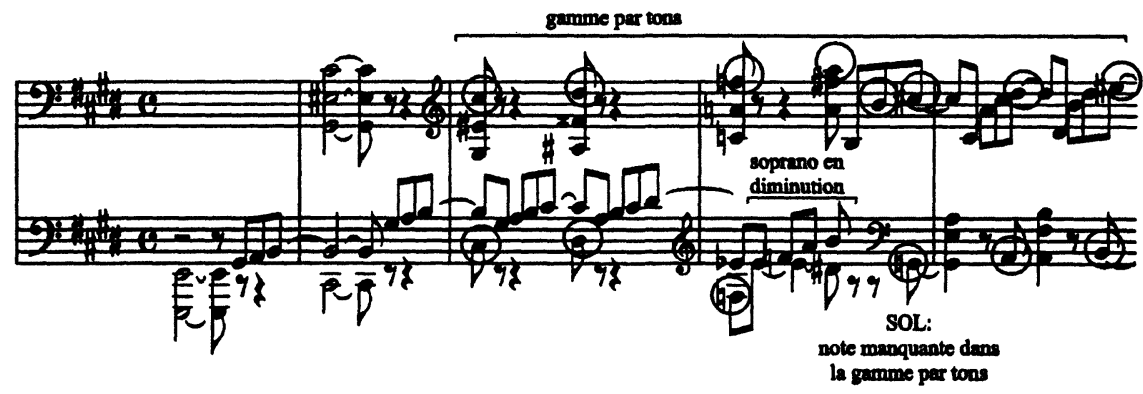

Exemple 23. Nocturne no 10 , mes. 62-66

directions tellement inédites que l'on peut parler d'un trait caractéristique du langage. Une coloration particulière se dégage tangiblement de tous les nocturnes en raison de la nature des enchaînements par tierces qui sont utilisés : ceux-ci ne comportent qu'une seule note commune (exemple 24). De ce fait, ils induisent nécessairement une fausse relation chromatique et élargissent le spectre des degrés aux douze demi-tons de la gamme chromatique.

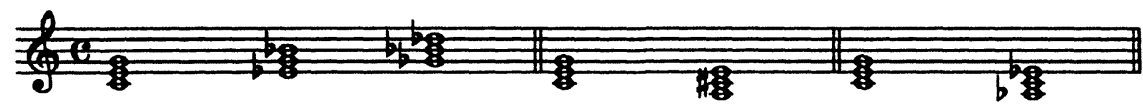

Exemple 24. Enchaînements par tierces avec une seule note commune

Nous allons maintenant observer dans quels contextes l'harmonie des médiantes est employée. Un premier cas est celui où une incise de médiantes, déstabilisatrice en contexte tonal, se produit en cours de phrase. On en retrouve un bon exemple dans le Nocturne $n^{o} 3$ (exemple 25) : les mesures 11 et 12 se résument à l'accord de mi majeur, alors que les fondamentales de la mesure précédente et de la suivante sont respectivement la bémol (I) et mi bémol (V). Par enharmonie, mi est en relation de tierce majeure avec la bémol. Même dans 
le cadre de la tonalité classique, ce mi pourrait être interprété comme un $f a$ bémol, sixième degré abaissé. Toutefois, le passage de la bémol à mi se fait de manière tellement directe, à cause de la présence d'une seule note commune (la bémol - sol dièse), que la référence aux fonctions tonales classique n'est plus tangible.
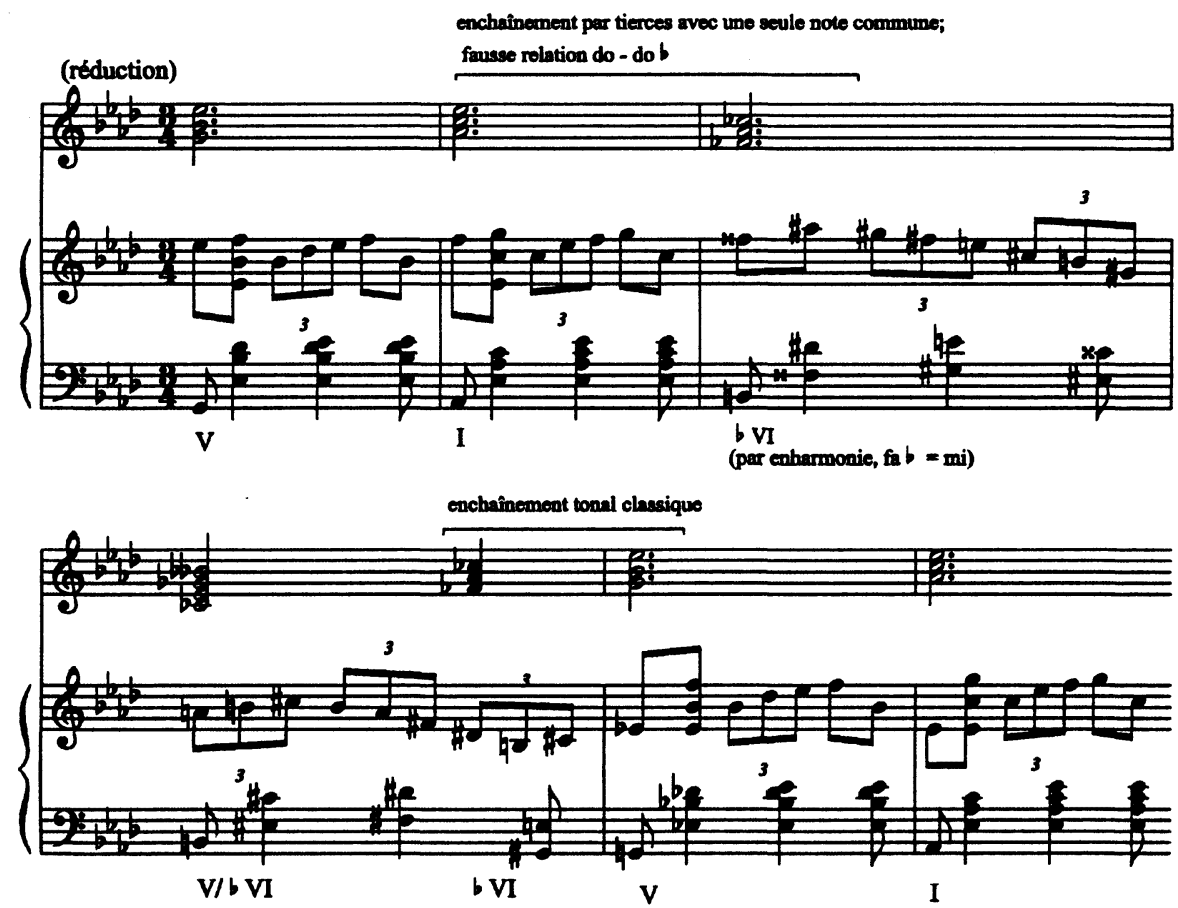

Exemple 25. Nocturne $n^{0} 3$, mes 9-14, accompagné d'une réduction

L'harmonie des médiantes est également employée en contexte d'oscillation, comme aux mesures 26-44 du Nocturne $n^{o} 5$ (exemple 26). Au départ, il y a une oscillation entre l'accord de septième de dominante sur $f a$ et l'accord parfait mineur de la bémol, qui est plutôt dissonante à cause de la fausse relation chromatique supplémentaire engendrée par la présence d'une septième. Une autre oscillation, moins dissonante cette fois, se produit ensuite entre si bémol majeur et ré bémol majeur. Finalement, tout le passage procède clairement par harmonie des médiantes, et échappe de ce fait aux usages de la tonalité classique. Toutefois, il est bien encadré par des fonctions nettement tonales, où la dominante joue son rôle structurant. On peut donc étendre à l'harmonie des médiantes l'affirmation de Johansen voulant que les éléments modaux soient contenus dans les bornes de la tonalité29. Une autre observation mérite d'être faite : la suggestion d'une relation dominante-tonique 
en ré (mes. 34-35), qui bifurque vers ré bémol, subordonne la dominante au jeu des médiantes, puisque ré est la médiante du ton de si bémol, tonalité affirmée par la cadence au ton principal qui clôt ce passage. Ainsi, Fauré instaure subtilement de nouvelles fonctions tonales au sein de son langage harmonique.
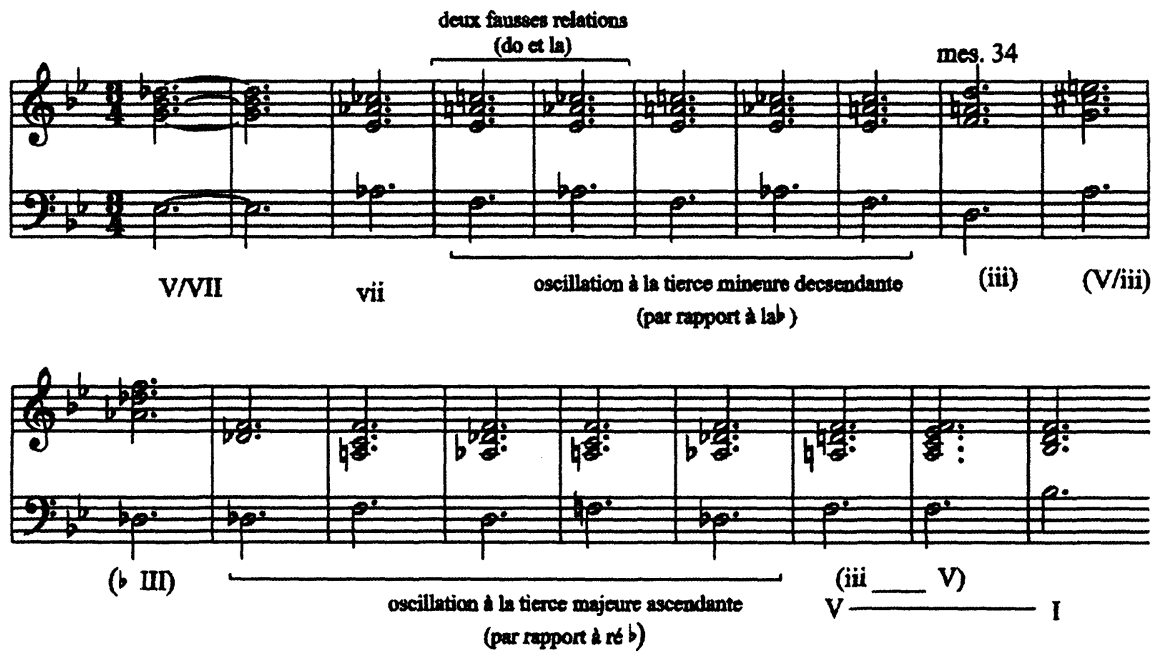

Exemple 26. Nocturne $n^{0} 5$, mes. 26-44 (réduction)

Lorsque l'harmonie des médiantes procède par tierces majeures, il en résulte une division symétrique de l'octave par enharmonie, comme à l'exemple 27, où les accords de sol dièse, $m i$, do et sol dièse s'enchaînent par tierces descendantes. La présence d'appoggiatures et d'un accord ornemental (entre parenthèses) vient masquer quelque peu la véritable nature de la progression.

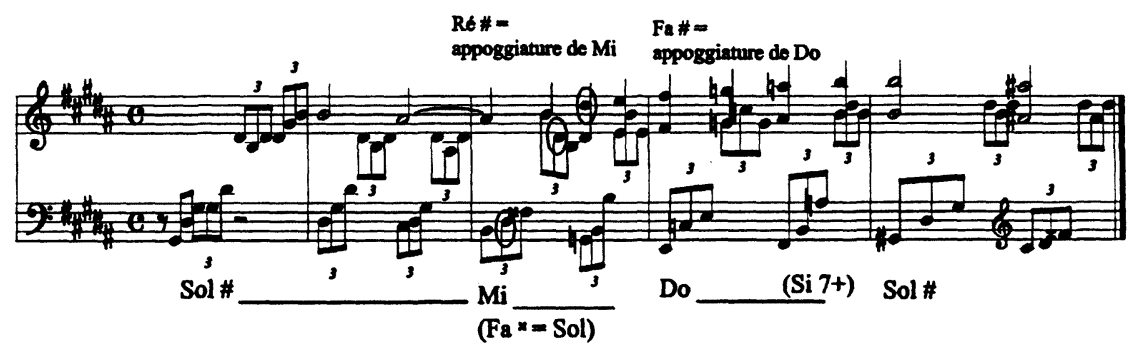

Exemple 27. Nocturne $n^{\circ} 13$, mes 55-59: division symétrique de l'octave par tierces majeures

Tout comme les tierces, les tritons génèrent des successions d'accords qui échappent à la tonalité usuelle. Le triton est parfois employé comme variante dans une succession en tierces (exemple 28). 


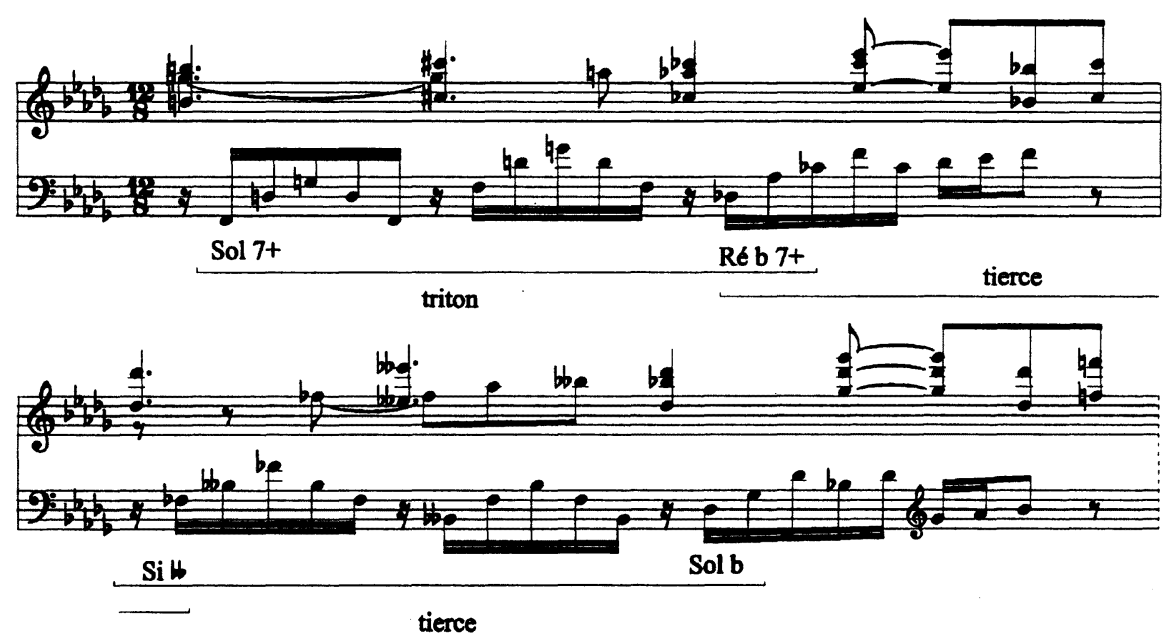

Exemple 28. Nocturne $n^{\circ} 8$, mes. 19-20

\section{LA LIBRE POLYPHONIE}

Une des conséquences de l'importance du contrepoint dans l'écriture fauréenne est l'apparition de procédés de libre polyphonie. À l'instar des polyphonistes de la Renaissance, pour lesquels l'accord n'est que le résultat des consonances entre des voix autonomes mélodiquement, on retrouve chez Fauré des passages qui s'expliquent aisément de manière linéaire, mais non par accords. La perception harmonique se brouille pendant que la conduite des voix demeure prévisible, notamment grâce au mouvement conjoint, réel ou octavié. En fait, il n'y a souvent pas de véritable mouvement harmonique, puisque les accords sont générés par la rencontre des voix. Les mesures 59-61 du Nocturne $n^{\circ} 6$ (exemple 29) offrent un tel passage, dans le contexte d'une incise avant une cadence sectionnelle.

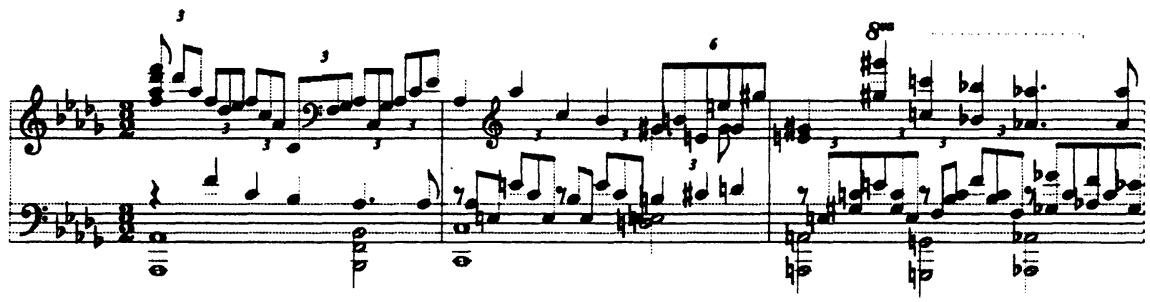

Réduction contrapuntique

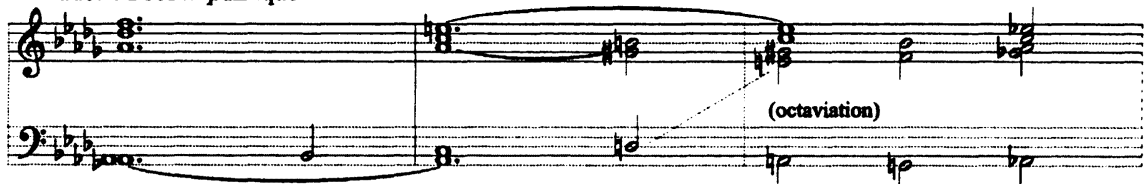

Exemple 29. Nocturne $n^{\circ} 6$, mes 59-61, accompagné d'une réécriture contrapuntique 
De plus, la conduite des voix habituelle subit d'importantes transformations. Si l'on compare le passage dans la partition avec sa réécriture contrapuntique, on observe une ligne conductrice certes conjointe, mais qui change de voix et qui est parfois octaviée. D'une certaine façon, cette technique fait penser à certains procédés d'orchestration de Mahler, avec des lignes d'abord continues qui, par la suite, changent d'instrument. Ainsi, le remodelage de la conduite des voix et du contrepoint effectué par Fauré s'inscrit dans les tendances novatrices de son époque.

\section{FORME ET ÉQUILIBRE TONAL}

Nous avons vu jusqu'ici comment l'harmonie tonale, avec ses cadences et sa dominante, parvenait à baliser des zones non tonales où de nouveaux paramètres harmoniques et contrapuntiques venaient enrichir le discours. Il faut maintenant préciser que l'harmonie par tierces joue parfois un rôle structurant du point de vue de la forme, bien qu'elle demeure subordonnée aux dominantes. Le tableau 2 indique comment interagissent les harmonies de médiantes et de dominantes dans la première partie du Nocturne $n^{0} 6$, tant dans la structure mélodique que dans la structure cadentielle. Autour de la tonalité principale, ré bémol majeur, des accords cadentiels se font entendre par enharmonie à la tierce supérieure $(m i)$ et inférieure $(l a)$. De plus, l'utilisation de l'échelle chromatique dans le choix des degrés permet de créer une équivoque entre la dominante, la bémol, et la médiante inférieure, la bécarre. Du point de vue des notes de sommet et de tombée, par contre, la tonique et la dominante sont généralement bien affirmées, sauf à l'avant-dernière phrase. Il est intéressant de noter comment l'instabilité harmonique locale est compensée par la stabilité globale du passage, qui n'est pas vraiment modulant.

Tableau 2. Harmonie des dominantes et harmonie des médiantes dans le Nocturne $n^{o} 6$ de Fauré

\begin{tabular}{|l|l|l|l|}
\hline Phrase & Mesures & \multicolumn{1}{|c|}{$\begin{array}{c}\text { Notes réelles de sommet } \\
\text { et de tombée }\end{array}$} & Accords de départ et d'arrivée \\
\hline 1 & $1-3$ & Ré bémol - ré bémol & Ré bémol - ré bémol \\
\hline 2 & $4-5$ & La bémol - mi bémol & La bémol - la bémol \\
\hline 3 & $6-7$ & La bémol - la bécarre & La bémol - la bécarre \\
\hline 4 & $7-10$ & Do dièse - ré bémol & La bécarre - ré bémol \\
\hline 5 & $11-12$ & Ré bémol - la bémol & Ré bémol - la bémol \\
\hline 6 & $13-14$ & Mi bécarre - si bécarre & La bécarre - si bécarre \\
\hline 7 & $15-18$ & Sol dièse - ré bémol & Mi bécarre - ré bémol \\
\hline
\end{tabular}

Du point de vue du parcours tonal global, le Nocturne $n^{\circ} 6$ ne s'écarte que momentanément de ré bémol (mode mixte), avec un bref passage modulant en la majeur ( $3^{\mathrm{e}}$ section), comme le démontre le tableau 3. Toutefois, cette $3^{e}$ section ne se termine pas par une cadence claire, ni ne s'affirme fortement en la majeur, ce qui renforce la sensation d'unitonalité au détriment du contraste tonal. 
Tableau 3. Plan tonal du Nocturne $n^{\circ} 6$

\begin{tabular}{|c|l|l|l|}
\hline Section & Mesures & \multicolumn{1}{|c|}{ Tonalité affirmées } & \multicolumn{1}{|c|}{$\begin{array}{c}\text { Tonalités moins affirmées } \\
\text { mais récurrentes }\end{array}$} \\
\hline 1 & $1-18$ & Ré bémol majeur & La et mi majeur \\
\hline 2 & $19-62$ & Do dièse mineur & \\
\hline 3 & $63-79$ & La majeur & Do majeur; modulation en do dièse \\
\hline 4 & $80-114$ & Do dièse & Parcours très instable \\
\hline 5 & $114-\mathrm{fin}$ & Ré bémol majeur & La et mi majeur \\
\hline
\end{tabular}

Les derniers nocturnes seront encore plus statiques dans le parcours tonal global, alors que les enchaînements d'accords deviendront plus flous. Ainsi, le Nocturne $n^{\circ} 9$ ne fait entendre aucune autre cadence que sur I ou V en si mineur.

\section{ÉCRITURE ET ESTHÉTIQUE}

À la lumière des exemples précédents, nous pouvons affirmer que la tonalité chez Fauré évolue vers un certain statisme harmonique. Localement, la notion de progression harmonique, au sens classique du terme, tend à disparaître au profit des enchaînements intervalliques directionnels ou des séquences contrapuntiques. Globalement, la notion de modulation s'estompe pour faire place à l'unitonalité. Ce sens de la zone tonale, qui se combine à des progressions estompées, peut être mis en parallèle avec nombre de procédés d'écriture impressionnistes, comme la pédale, l'ostinato et des successions d'accords parallèles ${ }^{30}$, même si Fauré conserve habilement les points de repère classiques pour les cadences et pour la conduite des voix.

On peut également déceler une évolution esthétique en transition entre le romantisme d'inspiration wagnérienne et le néoclassicisme des années 1920. La réserve expressive croissante qui enveloppe l'univers fauréen s'inscrit dans cette tendance. Techniquement, celle-ci se traduit par l'absence de la dominante et de sensible en cours de phrase, ce qui enlève de la directionnalité aux enchaînements, les rendant peu prévisibles et, de ce fait, ne créant pas d'attente tonale. On pourrait objecter que c'est en raison du genre, le nocturne, que le discours devient plus statique. Mais une analyse d'autres auvres du compositeur - mélodies et musique de chambre, notamment, qui fonctionnent de la même manière - démontrerait clairement la récurrence des procédés dans l'ensemble de la production fauréenne. D'ailleurs, les travaux récents de Caballero mettent en lumière comment Fauré adhère à l'esthétique de l'homogénéité ${ }^{31}$. Le statisme harmonique trouve un écho dans le traitement mélodique. Le compositeur élabore un mouvement entier sur un nombre restreint de cellules mélodico-rythmiques simples, subtilement altérées par des procédés usuels de contrepoint : transposition, renversement, rétrograde et permutation.

30 On retrouve notamment une bonne synthèse de ces procédés dans Ulehla, Contemporary Harmony, 159-248.

31 Carlo Caballero, Fauré and French Musical Aesthetics, coll. « Music in the Twentieth Century " (Cambridge : Cambridge University Press, 2001). 


\section{CONCLUSION}

Dans cet article, j'ai présenté les différents aspects du langage harmonique de Fauré selon une méthodologie relative à l'enseignement de l'écriture. En effet, le modèle de l'enseignement de la composition tonale passe par le développement organique de la forme (cadence, phrase, période, petite forme, etc.) ${ }^{32}$. Dans un contexte fauréen, certaines particularités doivent cependant être prises en compte. Tout d'abord, les diverses colorations de la dominante et de son environnement immédiat doivent faire l'objet d'une étude particulière. En outre, les divers procédés et contextes cadentiels ne se comprennent que lorsqu'ils sont mis en relation avec le type d'enchaînements employés dans la phrase. Basés sur la notion de substituts et d'équivoques, ces enchaînements doivent se construire à partir d'accords qui ne précisent pas trop la tonalité, notamment par l'évitement des dominantes et l'emploi de septièmes défonctionnalisées. Le degré de dissonance, généré tant par les notes étrangères que par des septièmes de toutes espèces, voire par des neuvièmes, sera maintenu de manière continue et cohérente.

Une fois que l'écriture de sections structurées en phrases est bien maîtrisée, la prochaine étape consiste à composer des sections aux contours moins définis, tant harmoniquement que formellement. Ces sections peuvent être d'une durée variable : brèves comme dans les premiers nocturnes, en alternance avec des sections structurées (comme dans les Nocturnes $n^{o s} 6$ et 7 ), ou occupant l'essentiel du discours, comme dans les derniers nocturnes. Ces sections moins définies seront néanmoins organisées à l'aide des nouvelles fonctions harmoniques développées par Fauré, notamment l'harmonie des médiantes avec une seule note commune, les progressions monointervalliques, séquentielles ou en libre polyphonie. L'étape finale dans l'apprentissage de l'harmonie fauréenne consiste en une recherche d'équilibre entre la stabilité tonale macroscopique et les contrastes microscopiques qui régissent l'enchaînement des accords. En fait, le but est d'en venir à une conception de l'harmonie fondée sur l'absence de progressions réelles, au profit d'une succession de zones et d'une prédominance de la ligne sur l'accord.

Si on la compare à l'harmonisation de chants donnés - une pratique surtout utile pour la conduite des voix - , ce type d'approche constitue une démarche plus complète et globale de l'harmonie qui, en outre, peut s'insérer dans une vision stylistique et esthétique de la composition. Tout langage harmonique comporte des implications non seulement à court terme, mais aussi à long terme. C'est pourquoi l'enseignement de l'harmonie ne peut être dissocié de l'apprentissage de la forme. C'est une solution vers laquelle convergent les pédagogues francophones qui étaient présents au colloque de la Société française d'analyse musicale (SFAM) de mars 2001, à Paris, lequel avait précisément pour thème l'enseignement de l'écriture.

Avant de terminer, je précise que le présent article ne prétend pas fournir une description exhaustive du langage fauréen. Bien conscient de la multipli-

32 À ce sujet, voir Arnold Schoenberg, Fundamentals of Musical Composition (Londres : Faber and Faber, 1967). 
cité des aspects de ce langage, j'ai voulu, pour des fins méthodologiques, me concentrer uniquement sur l'aspect harmonique de l'écriture. D'autres aspects importants pourront faire l'objet d'autres études, notamment le contrepoint, les motifs et les rapports entre la mélodie et l'harmonie. Du côté des recherches plus théoriques, il est vrai que la théorie harmonique riemannienne se rapproche à certains égards de l'utilisation de l'harmonie des médiantes chez Fauré; il serait intéressant d'étudier les correspondances qui existent entre les deux ${ }^{33}$. Si je n'ai pas développé cette approche, c'est que la théorie riemannienne n'a pas pour but d'expliquer les structures, mais plutôt de décrire et de classifier les accords. Enfin, je souhaite que le présent article soit le prélude à une réflexion plus vaste visant à intégrer le langage harmonique des années 1880-1920 dans les pratiques pédagogiques universitaires francophones, où l'enseignement de la théorie musicale passe encore beaucoup par l'écriture. Cette particularité francophone appelle un type de recherche qui n'exclut nullement la dimension analytique, mais qui la situe dans une finalité différente de celle des autres universités nord-américaines.

\section{Résumé}

Dans une analyse orientée vers des applications en écriture, l'auteur démontre comment Fauré intègre à la tonalité classique une conception nouvelle des fonctions harmoniques. Si la dominante conserve son rôle structurant en contexte cadentiel, la multiplication des dominantes défonctionnalisées rendent équivoques les progressions en cours de phrase. Comme autres procédés, on observe par endroits un usage « autonome » de la modalité, la séquence harmonique, l'harmonie des médiantes et un contrepoint qui prédomine de plus en plus sur l'harmonie; ces techniques viennent modifier considérablement, mais de l'intérieur, les caractéristiques du langage tonal. Au lieu de définir ces nouvelles caractéristiques par rapport à la tonalité, il est préférable de parler de nouvelles fonctions tonales. Celles-ci deviennent parties intégrantes du langage car elles conditionnent la structure harmonique globale.

\section{Abstract}

An analysis focused on stylistic applications reveals how Fauré incorporated a new concept of harmonic functions into the classical system of tonality. Although the dominant retains its role of giving structure to the cadence, the proliferation of dominants lacking precise function creates ambiguity in the progressions within the phrase. As for other methods, in places an "autonomous" use of the modality, of the harmonic sequence, and of the harmony of the mediants are noticeable, as well as a counterpoint that predominates more and more over the harmony. The above techniques modify deeply, but from inside 
the text, the characteristics of the tonal language. Rather than defining the new characteristics in terms of tonality, it is better to call them new tonal functions. Since they affect the whole of the harmonic structure these functions become part and parcel of the language. 